\title{
ANALYSIS OF A DYNAMIC CONTACT PROBLEM WITH FRICTION, DAMAGE AND ADHESION
}

Abstract. We study a dynamic contact problem for viscoelastic materials with damage. The contact is modelled with Tresca's friction law and a first order differential equation which describes adhesion effect of contact surfaces; the damage of the material is described by a function whose evolution is governed by a parabolic inclusion. Under appropriate assumptions, we provide a variational formulation of the mechanical problem and establish the existence and uniqueness of a weak solution.

1. Introduction. Processes of adhesion are important in many industrial settings, especially when a glue is added to prevent relative motion of the surfaces. For this industrial interest adhesive contact problems have recently received increased attention in the mathematical literature. An early attempt to study models of contact with adhesion was made in [8]-10]. Analysis and numerical simulations of frictionless contact problems with adhesion can be found in [4, [5], [15], [16]. In [4] the dynamic frictionless adhesive contact problem, with normal compliance condition, was modelled and analyzed; a fully discrete scheme was introduced and some numerical examples were included. The unilateral quasistatic contact problem with local friction and adhesion was studied in [5]; an existence result, for a friction coefficient small enough, was established. The main new idea in these papers is the introduction of the surface state variable $\beta$, the bonding field, which has values between 0 and 1 and measures the fractional density of

2010 Mathematics Subject Classification: 47J20, 49J40, 74M10, 74M15.

Key words and phrases: adhesion, dynamic process, viscoelastic materials, damage, Tresca's friction law.

Received 15 July 2016; revised 17 May 2018.

Published online 14 December 2018. 
active bonds. When $\beta=0$ there are no active bonds; when $\beta=1$ all the bonds are active; when $0<\beta<1$ partial adhesion takes place.

On the other hand, material damage, which may be caused by the growth of internal microcracks, appears in many applications of solids mechanics. Since it directly reduces the usefulness of the structures or components, the subject is important in design engineering. General models for damage were derived in [11, 12] from the virtual power principle. In these papers the evolution of the microscopic cracks which cause the damage is determined by a parabolic inclusion. In an isotropic and homogeneous elastic material, the damage function is defined by

$$
\zeta=\mathcal{E}_{\text {eff }} / \mathcal{E}_{\mathrm{Y}},
$$

where $\mathcal{E}_{\mathrm{Y}}$ is the Young modulus of the original material and $\mathcal{E}_{\text {eff }}$ is the current one. It follows that the damage function $\zeta$ has values between 0 and 1 . Recent modeling, analysis and numerical simulations of contact problems which include the evolution of material damage can be found in [3], [13], [17], [18, 22], 23] and references therein.

This paper is a continuation and an extension of [25]. There, the constitutive law was assumed to be viscoelastic; the quasistatic adhesive contact problem with Tresca's friction law was investigated and the rate of the bonding field was assumed to be irreversible. Here, the novelty consists in dealing with a dynamic contact problem with Tresca's friction law in which both adhesion and damage are taken into account. Moreover, the adhesion field is described by a general function which may change sign and allows rebonding after debonding, and the process is assumed to be with memory so that it depends on the bonding history. Also, we assume that the mechanical properties of the body are described by a nonlinear viscoelastic constitutive law with damage, such that the damage does not affect the viscosity of the material, but only its elastic behaviour. We derive a variational formulation of the mechanical problem for which we prove the existence of a unique weak solution, and obtain regularity results for the solution. The proof is based on the regularization method (see e.g. [6]), nonlinear evolution equations with monotone operators, a version of the Cauchy-Lipschitz theorem and the Banach fixed point theorem.

The rest of this paper is organized as follows. In Section 2 we present the notation and some preliminaries. Section 3 is dedicated to describing the mechanical problem and deriving its variational formulation. The main existence and uniqueness theorem is established in Section 4.

2. Notation and preliminaries. Here we introduce the notation we shall use and some preliminary materials. Let $\Omega$ be a bounded domain, in the numerical space $\mathbb{R}^{d}(d=2,3)$ of variables $x=\left(x_{1}, \ldots, x_{d}\right)$, with a Lipschitz boundary $\Gamma$. We denote by $\mathbb{S}^{d}$ the space of second order symmetric tensors 
on $\mathbb{R}^{d}$. We define the inner products and the corresponding norms on $\mathbb{R}^{d}$ and $\mathbb{S}^{d}$ by

$$
\begin{array}{lll}
u \cdot v=\sum_{i=1}^{d} u_{i} v_{i}, & |u|=\sqrt[2]{u \cdot u}, & \forall u, v \in \mathbb{R}^{d} ; \\
\sigma \cdot \xi=\sum_{1 \leq i, j \leq d} \sigma_{i j} \xi_{i j}, & |\sigma|=\sqrt[2]{\sigma \cdot \sigma}, & \forall \sigma, \xi \in \mathbb{S}^{d} .
\end{array}
$$

We introduce the spaces

$$
\begin{aligned}
H & =L^{2}\left(\Omega ; \mathbb{R}^{d}\right), \quad \mathcal{Q}=L^{2}\left(\Omega ; \mathbb{S}^{d}\right), \\
H_{1} & =\{u \in H ; \varepsilon(u) \in \mathcal{Q}\}, \\
\mathcal{Q}_{1} & =\{\sigma \in \mathcal{Q} ; \operatorname{Div} \sigma \in H\} .
\end{aligned}
$$

Here and below, $\varepsilon: H_{1} \rightarrow \mathcal{Q}$ is the deformation operator, defined by

$$
\varepsilon(u)=\frac{1}{2}\left(\nabla u+(\nabla u)^{T}\right), \quad \forall u \in H_{1},
$$

where $(\nabla u)^{T}$ is the transpose of the matrix $\nabla u$ which is defined by

$$
\nabla u=\left(\frac{\partial u_{i}}{\partial x_{j}}\right)_{1 \leq i, j \leq d},
$$

and Div : $\mathcal{Q}_{1} \rightarrow H$ is the divergence operator, defined by

$$
\operatorname{Div} \sigma=\left((\operatorname{Div} \sigma)_{i}\right)_{1 \leq i \leq d}=\left(\sum_{j=1}^{d} \frac{\partial \sigma_{i j}}{\partial x_{j}}\right)_{1 \leq i \leq d}, \quad \forall \sigma \in \mathcal{Q}_{1} .
$$

Note that $H, \mathcal{Q}, H_{1}, \mathcal{Q}_{1}$ are Hilbert spaces equipped with the respective canonical inner products

$$
\begin{aligned}
(u, v)_{H} & =\int_{\Omega} u \cdot v d x, \quad(\sigma, \tau)_{\mathcal{Q}}=\int_{\Omega} \sigma \cdot \tau d x, \\
(u, v)_{H_{1}} & =(u, v)_{H}+(\varepsilon(u), \varepsilon(v))_{\mathcal{Q}}, \\
(\sigma, \tau)_{\mathcal{Q}_{1}} & =(\operatorname{Div} \sigma, \operatorname{Div} \tau)_{H}+(\sigma, \tau)_{\mathcal{Q}}
\end{aligned}
$$

and the associated norms are denoted by $\|\cdot\|_{H},\|\cdot\|_{\mathcal{Q}},\|\cdot\|_{H_{1}},\|\cdot\|_{\mathcal{Q}_{1}}$.

Let $H_{\Gamma}=H^{1 / 2}\left(\Gamma ; \mathbb{R}^{d}\right)$ and let $\tilde{\gamma}: H_{1} \rightarrow H_{\Gamma}$ be the trace map. For every $v \in H_{1}$ we also write $v$ for the trace $\tilde{\gamma}(v)$ of $v$ on $\Gamma$, and for all $v \in H_{1}$ we denote by $v_{\nu}$ and $v_{\tau}$ the normal and tangential components of $v$ on the boundary $\Gamma$ :

$$
v_{\nu}=v \cdot \nu, \quad v_{\tau}=v-v_{\nu} \nu \quad \text { on } \Gamma,
$$

here and below $\nu$ represents the unit outward normal vector to $\Gamma$. In a similar manner, the normal and tangential components of a regular (say $C^{1}$ ) tensor field $\sigma$ are defined by

$$
\sigma_{\nu}=\sigma \nu \cdot \nu, \quad \sigma_{\tau}=\sigma \nu-\sigma_{\nu} \nu \quad \text { on } \Gamma
$$


moreover the following Green's formula holds:

$$
(\operatorname{Div} \sigma, v)_{H}+(\sigma, \varepsilon(v))_{\mathcal{Q}}=\int_{\Gamma} \sigma \nu \cdot v d a, \quad \forall v \in H_{1},
$$

where $d a$ is the surface measure element.

Next, for every real Banach space $\left(X,\|\cdot\|_{X}\right)$ and $T>0$, we denote by $C([0, T] ; X)$ and $C^{1}([0, T] ; X)$ the spaces of continuous and continuously differentiable functions from $[0, T]$ to $X$, and we use the standard notation for the spaces $L^{p}(0, T ; X)$ and $W^{k, p}(0, T ; X), p \in[1, \infty]$ and $k \geq 1$. We need the following result (see, e.g., [24, p. 60]).

Proposition 1. Let $\left(X,\|\cdot\|_{X}\right)$ be a real Banach space and let $F(t, \cdot)$ : $X \rightarrow X$ be an operator defined a.e. on $(0, T)$ which satisfies

(i) there exists $L_{F}>0$ such that $\|F(t, z)-F(t, y)\|_{X} \leq L_{F}\|z-y\|_{X}$, $\forall z, y \in X$, a.e. $t \in(0, T)$;

(ii) there exists $p \in[1, \infty]$ such that the mapping $t \mapsto F(t, z)$ is in $L^{p}(0, T ; X)$ for each $z \in X$.

Then, for each $z_{0} \in X$ there exists a unique function $z \in W^{1, p}(0, T ; X)$ such that

$$
\begin{aligned}
\dot{z}(t) & =F(t, z(t)), \quad \text { a.e. } t \in(0, T), \\
z(0) & =z_{0} .
\end{aligned}
$$

Here and everywhere in this paper, the dot above a variable denotes its derivative with respect to time.

Let $X$ and $Y$ be real Hilbert spaces such that $X$ is dense in $Y$ and the injection map is continuous; the space $Y$ is identified with its own dual and with a subspace of the dual $X^{*}$ of $X$, i.e. $X \subset Y \subset X^{*}$ is a Gelfand triplet. Denote by $\|\cdot\|_{X},\|\cdot\|_{Y},\|\cdot\|_{X^{*}}$ and $\langle\cdot, \cdot\rangle_{X^{*} \times X}$ the norm on the spaces $X, Y$, $X^{*}$ and the duality pairing between $X$ and $X^{*}$, respectively.

An operator $A: X \rightarrow X^{*}$ is said to be hemicontinuous if the real function $t \mapsto\langle A(u+t v), w\rangle_{X^{*} \times X}$ is continuous on $[0,1]$ for all $u, v, w \in X$, and monotone if $\langle A v-A w, v-w\rangle_{X^{*} \times X} \geq 0$ for all $v, w \in X$. The operator $A: X \rightarrow X^{*}$ is called pseudomonotone (see, e.g., [19]) if the following conditions are satisfied:

$$
\left\{\begin{aligned}
\text { (i) } & A \text { is bounded; } \\
\text { (ii) } & \text { if }\left(w_{n}\right) \subset X \text { with } w_{n} \rightarrow w \text { weakly in } X, \text { and } \\
& \lim \sup \left\langle A\left(w_{n}\right), w_{n}-w\right\rangle_{X^{*} \times X} \leq 0, \text { then } \\
& \lim \inf \left\langle A\left(w_{n}\right), w_{n}-v\right\rangle_{X^{*} \times X} \geq\langle A(w), w-v\rangle_{X^{*} \times X}, \forall v \in X .
\end{aligned}\right.
$$

We have the following result which may be found in [1, p. 140].

Proposition 2. Let $X \subset Y \subset X^{*}$ be a Gelfand triplet. Assume that $A: X \rightarrow X^{*}$ is a hemicontinuous and monotone operator and there are real 
constants $\mathcal{C}_{1}>0, \mathcal{C}_{2}$ and $\mathcal{C}_{3}>0$ such that

$$
\begin{array}{rlrl}
\langle A v, v\rangle_{X^{*} \times X} & \geq \mathcal{C}_{1}\|v\|_{X}^{2}+\mathcal{C}_{2}, & \forall v \in X, \\
\|A v\|_{X^{*}} \leq \mathcal{C}_{3}\left(\|v\|_{X}+1\right), & \forall v \in X .
\end{array}
$$

Then, given $w_{0} \in Y$ and $f \in L^{2}\left(0, T ; X^{*}\right)$, there exists a unique function $w$ which satisfies

$$
\begin{gathered}
w \in L^{2}(0, T ; X) \cap C([0, T] ; Y), \quad \dot{w} \in L^{2}\left(0, T ; X^{*}\right), \\
\dot{w}(t)+A w(t)=f(t) \quad \text { in } X^{*}, \text { a.e. } t \in(0, T), \\
w(0)=w_{0} .
\end{gathered}
$$

The following abstract result can be found in [2, p. 124].

Proposition 3. Let $X \subset Y \subset X^{*}$ be a Gelfand triplet and let $K$ be a nonempty, closed and convex set of $X$. Assume that $a(\cdot, \cdot): X \times X \rightarrow \mathbb{R}$ is a continuous and symmetric bilinear form and there are real constants $c_{2}>0$ and $c_{1}$ such that

$$
a(v, v)+c_{1}\|v\|_{\mathrm{Y}}^{2} \geq c_{2}\|v\|_{X}^{2}, \quad \forall v \in X .
$$

Then, for each $w_{0} \in K$ and each $f \in L^{2}(0, T ; Y)$, there exists a unique function $w \in W^{1,2}(0, T ; Y) \cap L^{2}(0, T ; X)$ such that

$$
\begin{aligned}
& w(t) \in K, \quad \forall t \in[0, T], \\
& \langle\dot{w}(t), v-w(t)\rangle_{X^{*} \times X}+a(w(t), v-w(t)) \geq(f(t), v-w(t))_{\mathrm{Y}} \text {, } \\
& \forall v \in K \text {, a.e. } t \in(0, T), \\
& w(0)=w_{0} .
\end{aligned}
$$

We end this section with the following Gronwall type inequality.

Proposition 4. Assume that $f, g:[a, b] \rightarrow \mathbb{R}$ are continuous functions which satisfy

$$
f(t) \leq g(t)+c \int_{a}^{t} f(s) d s, \quad \forall t \in[a, b],
$$

where $c>0$ is a constant. Then

$$
f(t) \leq g(t)+c \int_{a}^{t} g(s) \exp (c(t-s)) d s, \quad \forall t \in[a, b] .
$$

The proof can be found in [14, p. 162].

3. Problem statement. Assumptions. Variational formulation. The physical setting is as follows. A deformable body occupies a bounded domain $\Omega \subset \mathbb{R}^{d}$ (with $d=2,3$ ). The body is described by a nonlinear viscoelastic constitutive law with damage and the process is dynamic in the time interval of interest $[0, T]$. We assume that the boundary $\Gamma$ of the 
domain $\Omega$ is Lipschitz continuous, and it is partitioned into three disjoint measurable parts $\Gamma_{1}, \Gamma_{2}, \Gamma_{3}$ such that meas $\left(\Gamma_{1}\right)>0$. The body is clamped on $\Gamma_{1}$ and therefore the displacement field vanishes there, while volume forces of density $f_{0}$ act in $\Omega$, and surface tractions of density $f_{2}$ act on $\Gamma_{2}$. The contact is supposed to be bilateral, adhesive and governed by Tresca's friction law. To simplify the notation, we do not indicate explicitly the dependence of various functions on the spatial variable $x \in \Omega \cup \Gamma$. Under the above assumptions, the classical formulation of our problem is the following.

Problem 5. Find a displacement field $u: \Omega \times[0, T] \rightarrow \mathbb{R}^{d}$, a stress field $\sigma: \Omega \times[0, T] \rightarrow \mathbb{S}^{d}$, a damage field $\zeta: \Omega \times[0, T] \rightarrow \mathbb{R}$ and a bonding field $\beta: \Gamma_{3} \times[0, T] \rightarrow \mathbb{R}$ such that

$$
\begin{aligned}
& \sigma=\mathcal{A} \varepsilon(\dot{u})+\mathcal{B}(\varepsilon(u), \zeta) \quad \text { in } \Omega \times(0, T), \\
& \dot{\zeta}-\kappa \Delta \zeta+\partial I_{[0,1]}(\zeta) \ni \mathcal{G}(\varepsilon(u), \zeta) \quad \text { in } \Omega \times(0, T) \text {, } \\
& \rho \ddot{u}=\operatorname{Div} \sigma+f_{0} \\
& u=0 \\
& \sigma \nu=f_{2} \\
& \frac{\partial \zeta}{\partial \nu}=0 \\
& \text { in } \Omega \times(0, T) \text {, } \\
& \text { on } \Gamma_{1} \times(0, T) \text {, } \\
& \text { on } \Gamma_{2} \times(0, T) \text {, } \\
& \text { on } \Gamma \times(0, T) \text {, } \\
& u_{\nu}=0 \\
& \text { on } \Gamma_{3} \times(0, T) \text {, } \\
& \left\{\begin{array}{l}
\left|\sigma_{\tau}+p_{\tau}\left(\beta, u_{\tau}\right)\right| \leq g_{b}, \\
\left|\sigma_{\tau}+p_{\tau}\left(\beta, u_{\tau}\right)\right|<g_{b} \Rightarrow \dot{u}_{\tau}=0, \\
\left|\sigma_{\tau}+p_{\tau}\left(\beta, u_{\tau}\right)\right|=g_{b} \Rightarrow \\
\quad \exists \lambda \geq 0: \sigma_{\tau}+p_{\tau}\left(\beta, u_{\tau}\right)=-\lambda \dot{u}_{\tau}
\end{array}\right. \\
& \dot{\beta}=H_{\mathrm{ad}}\left(\beta, \theta_{\beta},\left|R_{\tau}\left(u_{\tau}\right)\right|\right) \\
& \text { on } \Gamma_{3} \times(0, T) \text {, } \\
& \beta(0)=\beta_{0} \\
& \text { on } \Gamma_{3} \text {, } \\
& \zeta(0)=\zeta_{0} \\
& \text { in } \Omega \text {, } \\
& \text { (i) } u(0)=u_{0} \text {, (ii) } \dot{u}(0)=v_{0} \\
& \text { on } \Gamma_{3} \times(0, T) \text {, }
\end{aligned}
$$

Equation (6) represents the viscoelastic constitutive law with damage in which $\varepsilon$ denotes the linearized strain tensor, $\mathcal{A}$ is the viscosity operator and $\mathcal{B}$ is the elasticity operator. Here we assume that the damage affects only the elastic behaviour of the material, and therefore $\mathcal{B}$ is a function of the strain and the damage field. (7) is a parabolic differential inclusion which describes the evolution of the damage field, where $\Delta$ denotes the Laplace operator, $\kappa>0$ is the microcrack diffusion constant and $\mathcal{G}$ is the damage 
source function. The indicator function $I_{[0,1]}: \mathbb{R} \rightarrow(-\infty, \infty]$ is given by

$$
I_{[0,1]}(s)= \begin{cases}0 & \text { if } s \in[0,1] \\ \infty & \text { otherwise. }\end{cases}
$$

The subdifferential of $I_{[0,1]}$ at $s$ is the set

$$
\partial I_{[0,1]}(s)=\left\{r \in \mathbb{R} ; I_{[0,1]}(z)-I_{[0,1]}(s) \geq r(z-s), \forall z \in \mathbb{R}\right\}, \quad \forall s \in \mathbb{R},
$$
i.e.

$$
\partial I_{[0,1]}(s)= \begin{cases}(-\infty, 0] & \text { if } s=0, \\ 0 & \text { if } s \in(0,1), \\ {[0, \infty)} & \text { if } s=1, \\ \emptyset & \text { otherwise. }\end{cases}
$$

Therefore the subdifferential term $\partial I_{[0,1]}(\zeta)$ in $(7)$ guarantees that $\zeta$ is restricted to values between 0 and 1 ; when $\zeta=1$ the material is damage-free and has its full capacity; when $\zeta=0$ the material is completely damaged; when $0<\zeta<1$ there is partial additional damage. Equation (8) is the dynamic equation of motion where $\rho$ is the mass density. Equations (9)-(10) are the displacement-traction boundary conditions where $\sigma \nu$ represents the Cauchy stress vector. Equation (11) means that the normal derivative of $\zeta$, denoted by $\partial \zeta / \partial \nu$, vanishes on $\Gamma$. Therefore, there is no influx of microcracks across the boundary. Conditions (12)-(13) represent the bilateral contact with Tresca's friction law in which adhesion is taken into account and $g_{b}$ is a friction bound. Here, $p_{\tau}$ is a general prescribed function. In particular, we may consider the case

$$
p_{\tau}(\beta, v)= \begin{cases}q_{\tau}(\beta) v & \text { if } 0 \leq|v| \leq L, \\ q_{\tau}(\beta) L v /|v| & \text { if }|v|>L,\end{cases}
$$

where $L>0$ is a limit bound constant and $q_{\tau}$ is nonnegative tangential stiffness function (see, e.g., [21]). In [25] the following form of $q_{\tau}$ has been employed:

$$
q_{\tau}(\beta)=c_{\tau} \beta^{2} \quad \text { on } \Gamma_{3} \times(0, T),
$$

where $c_{\tau}$ is a given positive material parameter. Equation (14) represents the evolution of the bonding field described by a general function $H_{\mathrm{ad}}$ which may change sign. This condition implies that cycles of rebonding after debonding may take place (see [16], 22] for details). Moreover, the process depends on the bonding history, which we denote by

$$
\theta_{\beta}(t, x)=\int_{0}^{t} \beta(s, x) d s .
$$


Here and below, $R_{\tau}: \mathbb{R}^{d} \rightarrow \mathbb{R}^{d}$ is a truncation operator defined by

$$
R_{\tau}(v)= \begin{cases}v & \text { if } 0 \leq|v| \leq L, \\ L v /|v| & \text { if }|v|>L .\end{cases}
$$

The introduction of the operator $R_{\tau}$ is motivated by the mathematical arguments where $L>0$ is a characteristic length of the bond, beyond which there is no any additional traction. Clearly, $R_{\tau}$ satisfies

$$
\begin{cases}\left|R_{\tau}(v)\right| \leq L, & \forall v \in \mathbb{R}^{d}, \\ || R_{\tau}(w)|-| R_{\tau}(v)|| \leq|w-v|, & \forall w, v \in \mathbb{R}^{d} .\end{cases}
$$

An example of the adhesion rate function $H_{\mathrm{ad}}$ is

$$
H_{\mathrm{ad}}(\beta, r)=-\left(c_{\tau} \beta r^{2}-\epsilon_{a}\right)_{+} \quad \text { on } \Gamma_{3} \times(0, T),
$$

where $c_{\tau}, \epsilon_{a}$ are given positive material parameters and $(\alpha)_{+}$denotes the positive part of $\alpha$, that is, $(\alpha)_{+}=\max \{\alpha, 0\}$. Since $\dot{\beta} \leq 0$, the process is irreversible and once debonding occurs, bonding cannot be reestablished (see, e.g., 44, 25]). Another example, in which $H_{\text {ad }}$ depends on all three variables, is

$$
H_{\mathrm{ad}}\left(\beta, \theta_{\beta}, r\right)=-\gamma_{1} \beta r^{2}+\gamma_{2} \frac{\beta_{+}(1-\beta)_{+}}{1+d_{*}\left(\theta_{\beta}\right)^{2}},
$$

where $\gamma_{1}, \gamma_{2}$ are given positive material parameters and $d_{*}>0$ is the history weight factor (see, e.g., 7], [16, [22]). Finally, (15)-(17) are the initial conditions.

To obtain a variational formulation of the mechanical problem, we introduce the space $V$ and the convex set $\mathcal{K}$ defined by

$$
\begin{aligned}
& V=\left\{v \in H_{1} ; v=0 \text { on } \Gamma_{1}, v_{\nu}=0 \text { on } \Gamma_{3}\right\}, \\
& \mathcal{K}=\left\{\zeta \in H^{1}(\Omega) ; 0 \leq \zeta \leq 1, \text { a.e. } x \in \Omega\right\} .
\end{aligned}
$$

Since meas $\left(\Gamma_{1}\right)>0$, Korn's inequality holds:

$$
C_{K}\|v\|_{H_{1}} \leq\|\varepsilon(v)\|_{\mathcal{Q}}, \quad \forall v \in V,
$$

where $C_{K}>0$ is a positive constant depending only on $\Omega$ and $\Gamma_{1}$. A proof of Korn's inequality can be found, for instance, in [20, p. 79]. Over the space $V$, we consider the inner product given by

$$
(u, v)_{V}=(\varepsilon(u), \varepsilon(v))_{\mathcal{Q}}, \quad \forall u, v \in V
$$

and let $\|\cdot\|_{V}$ be the associated norm. It follows from Korn's inequality (19) that $\|\cdot\|_{H_{1}}$ and $\|\cdot\|_{V}$ are equivalent norms on $V$. Therefore $\left(V,(\cdot, \cdot)_{V}\right)$ is a real Hilbert space. Moreover, by the Sobolev trace theorem, there exists a positive constant $c_{0}$ depending only on the domain $\Omega, \Gamma_{1}$ and $\Gamma_{3}$ such that

$$
\|v\|_{L^{2}\left(\Gamma_{3} ; \mathbb{R}^{d}\right)} \leq c_{0}\|v\|_{V}, \quad \forall v \in V .
$$


In the study of the mechanical problem (6) -17 ) we consider the following assumptions. We assume that the viscosity operator $\mathcal{A}: \Omega \times \mathbb{S}^{d} \rightarrow \mathbb{S}^{d}$ satisfies

(i) there exists $m_{\mathcal{A}}>0$ such that

$$
\begin{aligned}
& \left(\mathcal{A}\left(x, \varepsilon_{1}\right)-\mathcal{A}\left(x, \varepsilon_{2}\right)\right) \cdot\left(\varepsilon_{1}-\varepsilon_{2}\right) \geq m_{\mathcal{A}}\left|\varepsilon_{1}-\varepsilon_{2}\right|^{2}, \\
& \forall \varepsilon_{1}, \varepsilon_{2} \in \mathbb{S}^{d} \text {, a.e. } x \in \Omega
\end{aligned}
$$

(ii) there exists $L_{\mathcal{A}}>0$ such that

$$
\begin{aligned}
& \left|\mathcal{A}\left(x, \varepsilon_{1}\right)-\mathcal{A}\left(x, \varepsilon_{2}\right)\right| \leq L_{\mathcal{A}}\left|\varepsilon_{1}-\varepsilon_{2}\right|, \\
& \forall \varepsilon_{1}, \varepsilon_{2} \in \mathbb{S}^{d} \text {, a.e. } x \in \Omega ;
\end{aligned}
$$

(iii) the mapping $x \mapsto \mathcal{A}(x, \varepsilon)$ is Lebesgue measurable on $\Omega$ for any $\varepsilon \in \mathbb{S}^{d}$;

(iv) the mapping $x \mapsto \mathcal{A}\left(x, 0_{\mathbb{S}^{d}}\right)$ belongs to $\mathcal{Q}$.

We assume that the operator $\mathcal{B}: \Omega \times \mathbb{S}^{d} \times \mathbb{R} \rightarrow \mathbb{S}^{d}$ satisfies

(i) there exists $L_{\mathcal{B}}>0$ such that

$$
\left\{\begin{array}{l}
\left|\mathcal{B}\left(x, \varepsilon_{1}, \xi_{1}\right)-\mathcal{B}\left(x, \varepsilon_{2}, \xi_{1}\right)\right| \leq L_{\mathcal{B}}\left(\left|\varepsilon_{1}-\varepsilon_{2}\right|+\left|\xi_{1}-\xi_{2}\right|\right), \\
\quad \forall \varepsilon_{1}, \varepsilon_{2} \in \mathbb{S}^{d}, \forall \xi_{1}, \xi_{2} \in \mathbb{R}, \text { a.e. } x \in \Omega ; \\
\text { (ii) the mapping } x \mapsto \mathcal{B}(x, \varepsilon, \xi) \text { is Lebesgue measurable on } \Omega, \\
\quad \forall \varepsilon \in \mathbb{S}^{d}, \forall \xi \in \mathbb{R} ; \\
\text { (iii) the mapping } x \mapsto \mathcal{B}\left(x, 0_{\mathbb{S}^{d}}, 0_{\mathbb{R}}\right) \text { belongs to } \mathcal{Q} .
\end{array}\right.
$$

We assume that the damage source function $\mathcal{G}: \Omega \times \mathbb{S}^{d} \times \mathbb{R} \rightarrow \mathbb{R}$ satisfies

(i) there exists $L_{\mathcal{G}}>0$ such that

$$
\begin{aligned}
&\left|\mathcal{G}\left(x, \varepsilon_{1}, \xi_{1}\right)-\mathcal{G}\left(x, \varepsilon_{2}, \xi_{1}\right)\right| \leq L_{\mathcal{G}}\left(\left|\varepsilon_{1}-\varepsilon_{2}\right|+\left|\xi_{1}-\xi_{2}\right|\right), \\
& \forall \varepsilon_{1}, \varepsilon_{2} \in \mathbb{S}^{d}, \forall \xi_{1}, \xi_{2} \in \mathbb{R}, \text { a.e. } x \in \Omega ; \\
& \text { (ii) the mapping } x \mapsto \mathcal{G}(x, \varepsilon, \xi) \text { is Lebesgue measurable on } \Omega, \\
& \quad \forall \varepsilon \in \mathbb{S}^{d}, \forall \xi \in \mathbb{R} ; \\
& \text { (iii) the mapping } x \mapsto \mathcal{G}\left(x, 0_{\mathbb{S}^{d}}, 0_{\mathbb{R}}\right) \text { belongs to } L^{2}(\Omega) .
\end{aligned}
$$

We assume that the tangential contact function $p_{\tau}: \Gamma_{3} \times \mathbb{R} \times \mathbb{R}^{d} \rightarrow \mathbb{R}^{d}$ satisfies 
(i) there exists $L_{\tau}>0$ such that $\left|p_{\tau}\left(x, \beta_{1}, r_{1}\right)-p_{\tau}\left(x, \beta_{2}, r_{2}\right)\right| \leq L_{\tau}\left(\left|\beta_{1}-\beta_{2}\right|+\left|r_{1}-r_{2}\right|\right)$, $\forall \beta_{1}, \beta_{2} \in[0,1], \forall r_{1}, r_{2} \in \mathbb{R}^{d}$, a.e. $x \in \Gamma_{3} ;$

(ii) $r \cdot \nu(x)=0 \Rightarrow p_{\tau}(x, \beta, r) \cdot \nu(x)=0$, $\forall \beta \in \mathbb{R}, \forall r \in \mathbb{R}^{d}$, a.e $x \in \Gamma_{3} ;$

(iii) the mapping $x \mapsto p_{\tau}(x, \beta, r)$ is Lebesgue measurable on $\Gamma_{3}$ $\forall \beta \in \mathbb{R}, \forall r \in \mathbb{R}^{d} ;$

(iv) the mapping $x \mapsto p_{\tau}\left(x, 0_{\mathbb{R}}, 0_{\mathbb{R}^{d}}\right)$ belongs to $L^{2}\left(\Gamma_{3} ; \mathbb{R}^{d}\right)$.

As in [22], the adhesion rate function $H_{\mathrm{ad}}: \Gamma_{3} \times \mathbb{R} \times \mathbb{R} \times[0, L] \rightarrow \mathbb{R}$ is assumed to satisfy

(i) there exists $L_{H_{\text {ad }}}>0$ such that $\left|H_{\text {ad }}\left(x, \beta_{1}, z, r\right)-H_{\text {ad }}\left(x, \beta_{2}, z, r\right)\right| \leq L_{H_{\text {ad }}}\left|\beta_{1}-\beta_{2}\right|$, a.e. $x \in \Gamma_{3}, \forall \beta_{1}, \beta_{2}, z \in \mathbb{R}, \forall r \in[0, L]$;

(ii) $\left|H_{\mathrm{ad}}\left(x, \beta_{1}, z_{1}, r_{1}\right)-H_{\mathrm{ad}}\left(x, \beta_{2}, z_{2}, r_{2}\right)\right|$ $\leq L_{H_{\mathrm{ad}}}\left(\left|\beta_{1}-\beta_{2}\right|+\left|z_{1}-z_{2}\right|+\left|r_{1}-r_{2}\right|\right)$ $\forall \beta_{1}, \beta_{2} \in[0,1], \forall z_{1}, z_{2} \in \mathbb{R}, \forall r_{1}, r_{2} \in[0, L]$, a.e. $x \in \Gamma_{3} ;$

$\left\{\right.$ (iii) the mapping $x \rightarrow H_{\text {ad }}(x, \beta, z, r)$ is measurable on $\Gamma_{3}$, $\forall \beta, z \in \mathbb{R}, \forall r \in[0, L]$

(iv) the mapping $(\beta, z, r) \rightarrow H_{\mathrm{ad}}(x, \beta, z, r)$ is continuous on $\mathbb{R} \times \mathbb{R} \times[0, L]$, a.e. $x \in \Gamma_{3} ;$

(v) $H_{\mathrm{ad}}(x, 0, z, r)=0, \forall z \in \mathbb{R}, \forall r \in[0, L]$, a.e. $x \in \Gamma_{3}$;

(vi) $H_{\mathrm{ad}}(x, \beta, z, r) \geq 0, \forall \beta \leq 0, \forall z \in \mathbb{R}, \forall r \in[0, L]$, a.e. $x \in \Gamma_{3}$, $H_{\mathrm{ad}}(x, \beta, z, r) \leq 0, \forall \beta \geq 1, \forall z \in \mathbb{R}, \forall r \in[0, L]$, a.e. $x \in \Gamma_{3}$.

We suppose that the mass density satisfies

(26) $\rho \in L^{\infty}(\Omega)$ and there exists $\rho_{*}>0$ such that $\rho(x) \geq \rho_{*}$ a.e. $x \in \Omega$.

Also, we suppose that the friction bound function $g_{b}: \Gamma_{3} \rightarrow \mathbb{R}^{+}$satisfies

$$
g_{b} \in L^{\infty}\left(\Gamma_{3}\right) .
$$

The body forces and surface tractions have the regularity:

$$
f_{0} \in L^{2}(0, T ; H), \quad f_{2} \in L^{2}\left(0, T ; L^{2}\left(\Gamma_{2} ; \mathbb{R}^{d}\right)\right) .
$$

Finally, we assume that the initial data satisfy

$$
\begin{gathered}
\beta_{0} \in L^{\infty}\left(\Gamma_{3}\right), \quad 0 \leq \beta_{0}(x) \leq 1, \quad \text { a.e. } x \in \Gamma_{3}, \\
\zeta_{0} \in \mathcal{K},
\end{gathered}
$$
(i) $u_{0} \in V$,
(ii) $v_{0} \in H$. 
Next, we define a modified inner product on the Hilbert space $H$ by $(32)$

$$
((u, w))_{H}=(\rho u, w)_{H}, \quad \forall u, w \in H,
$$

and let $\|\cdot\|_{H}$ be the associated norm, i.e.

$$
\|w\|_{H}=\left[(\rho w, w)_{H}\right]^{1 / 2}, \quad \forall w \in H .
$$

It follows from (26) and (33) that $\|\cdot\|_{H}$ and $\|\cdot\|_{H}$ are equivalent norms on $H$. Moreover, the inclusion mapping of $\left(V,\|\cdot\|_{V}\right)$ into $\left(H,\|\cdot\|_{H}\right)$ is continuous and dense. Identifying $H$ with its own dual, we can write the Gelfand triplet

$$
V \subset H \subset V^{*} \text {. }
$$

We use $\langle,\rangle_{V^{*} \times V}$ to represent the duality pairing between $V^{*}$ and $V$. Then

$$
\langle v, w\rangle_{V^{*} \times V}=((v, w))_{H}, \quad \forall v \in H, \forall w \in V .
$$

Using Riesz's theorem, from 28 we can define $f \in L^{2}(0, T ; V)$ by

$$
(f(t), w)_{V}=\int_{\Omega} f_{0}(t) \cdot w d x+\int_{\Gamma_{2}} f_{2}(t) \cdot w d a, \quad \forall w \in V, \text { a.e. } t \in(0, T) .
$$

Below, we use the functionals $j_{\tau}: V \rightarrow \mathbb{R}$ and $j_{\text {ad }}: L^{\infty}\left(\Gamma_{3}\right) \times V \times V \rightarrow \mathbb{R}$ defined by

$$
\begin{aligned}
j_{\tau}(w) & =\int_{\Gamma_{3}} g_{b}\left|w_{\tau}\right| d a, \quad \forall w \in V, \\
j_{\mathrm{ad}}(\beta, v, w) & =\int_{\Gamma_{3}} p_{\tau}\left(\beta, v_{\tau}\right) \cdot w_{\tau} d a, \quad \forall(\beta, v, w) \in L^{\infty}\left(\Gamma_{3}\right) \times V \times V .
\end{aligned}
$$

Thanks to (24) and (20), there exists $L_{\text {ad }}>0$ such that

$$
\begin{aligned}
& \left|j_{\mathrm{ad}}\left(\beta_{1}, u_{1}, w\right)-j_{\mathrm{ad}}\left(\beta_{2}, u_{2}, w\right)\right| \\
& \leq L_{\mathrm{ad}}\left(\left\|\beta_{1}-\beta_{2}\right\|_{L^{2}\left(\Gamma_{3}\right)}+\left\|u_{1}-u_{2}\right\|_{V}\right)\|w\|_{V}, \quad \forall u_{1}, u_{2}, w \in V, \\
& \forall \beta_{1}, \beta_{2} \in L^{\infty}\left(\Gamma_{3}\right), 0 \leq \beta_{1}, \beta_{2} \leq 1, \text { a.e. } x \in \Gamma_{3} .
\end{aligned}
$$

We turn now to derive a variational formulation of the mechanical problem (6)-(17). To this end, assume that $(u, \sigma, \zeta, \beta)$ are smooth functions satisfying (6)-(17) and let $w \in V$ and $t \in[0, T]$. Using (8), (32), (34) and Green's formula (1), we obtain

$$
\langle\ddot{u}(t), w\rangle_{V^{*} \times V}+(\sigma(t), \varepsilon(w))_{\mathcal{Q}}-\int_{\Omega} f_{0}(t) \cdot w d x=\int_{\Gamma} \sigma(t) \nu \cdot w d a,
$$

and by $(10)$ we find

$$
\int_{\Gamma} \sigma(t) \nu \cdot w d a=\int_{\Gamma_{2}} f_{2}(t) \cdot w d a+\int_{\Gamma_{3}} \sigma_{\tau}(t) \cdot w_{\tau} d a .
$$


Then (39), (40) and (35) lead to

$$
\begin{aligned}
\langle\ddot{u}(t), w-\dot{u}(t)\rangle_{V^{*} \times V}+(\sigma(t), \varepsilon(w)-\varepsilon(\dot{u}(t)))_{\mathcal{Q}} & \\
& -\int_{\Gamma_{3}} \sigma_{\tau}(t) \cdot\left(w_{\tau}-\dot{u}_{\tau}(t)\right) d a=(f(t), w-\dot{u}(t))_{V} .
\end{aligned}
$$

On the other hand,

$$
-\int_{\Gamma_{3}}\left(\sigma_{\tau}(t)+p_{\tau}\left(\beta(t), u_{\tau}(t)\right)\right) \cdot w_{\tau} d a \leq \int_{\Gamma_{3}}\left|\sigma_{\tau}(t)+p_{\tau}\left(\beta(t), u_{\tau}(t)\right)\right|\left|w_{\tau}\right| d a,
$$

and taking into account the boundary condition (13), we deduce that

$$
\begin{aligned}
-\int_{\Gamma_{3}} \sigma_{\tau}(t) \cdot w_{\tau} d a \leq & \int_{\Gamma_{3}}\left|\sigma_{\tau}(t)+p_{\tau}\left(\beta(t), u_{\tau}(t)\right)\right|\left|w_{\tau}\right| d a \\
& +\int_{\Gamma_{3}} p_{\tau}\left(\beta(t), u_{\tau}(t)\right) \cdot w_{\tau} d a \\
\leq & \int_{\Gamma_{3}} g_{b}\left|w_{\tau}\right| d a+\int_{\Gamma_{3}} p_{\tau}\left(\beta(t), u_{\tau}(t)\right) \cdot w_{\tau} d a .
\end{aligned}
$$

Also, using (13) we obtain

$$
\begin{aligned}
\int_{\Gamma_{3}}\left(\sigma_{\tau}(t)+p_{\tau}\left(\beta(t), u_{\tau}(t)\right)\right) \cdot \dot{u}_{\tau}(t) d a & =-\int_{\Gamma_{3}}\left|\sigma_{\tau}(t)+p_{\tau}\left(\beta(t), u_{\tau}(t)\right)\right|\left|\dot{u}_{\tau}(t)\right| d a \\
& =-\int_{\Gamma_{3}} g_{b}\left|\dot{u}_{\tau}(t)\right| d a,
\end{aligned}
$$

which gives

$$
\int_{\Gamma_{3}} \sigma_{\tau}(t) \cdot \dot{u}_{\tau}(t) d a=-\int_{\Gamma_{3}} g_{b}\left|\dot{u}_{\tau}(t)\right| d a-\int_{\Gamma_{3}} p_{\tau}\left(\beta(t), u_{\tau}(t)\right) \cdot \dot{u}_{\tau}(t) d a,
$$

and keeping in mind 42 and (36)- 37 , we find

$$
\begin{aligned}
& -\int_{\Gamma_{3}} \sigma_{\tau}(t) \cdot\left(w_{\tau}-\dot{u}_{\tau}(t)\right) d a \\
& \quad \leq j_{\tau}(w)-j_{\tau}(\dot{u}(t))+j_{\mathrm{ad}}(\beta(t), u(t), w)-j_{\mathrm{ad}}(\beta(t), u(t), \dot{u}(t)) .
\end{aligned}
$$

Now, using integration by parts and applying condition (11) we get

$$
-(\Delta \zeta(t), \psi)_{L^{2}(\Omega)}=(\nabla \zeta(t), \nabla \psi)_{L^{2}\left(\Omega, \mathbb{R}^{d}\right)}, \quad \forall \psi \in H^{1}(\Omega) .
$$

Moreover, from (7) one has

$$
\begin{aligned}
(\dot{\zeta}(t)-\kappa \Delta \zeta(t))(\psi-\zeta(t))+I_{[0,1]}(\psi)-I_{[0,1]}(\zeta(t)) & \\
& \geq \mathcal{G}(\varepsilon(u(t)), \zeta(t))(\psi-\zeta(t)), \quad \forall \psi \in H^{1}(\Omega), \text { a.e. } x \in \Omega,
\end{aligned}
$$


and since $0 \leq \zeta(t) \leq 1$, we obtain

$$
\begin{aligned}
(\dot{\zeta}(t), \psi-\zeta(t))_{L^{2}(\Omega)} & -\kappa(\Delta \zeta(t), \psi-\zeta(t))_{L^{2}(\Omega)} \\
\geq & (\mathcal{G}(\varepsilon(u(t)), \zeta(t)), \psi-\zeta(t))_{L^{2}(\Omega)}, \quad \forall \psi \in \mathcal{K} .
\end{aligned}
$$

Finally, using (41), 43)-45) and keeping in mind (14)-(17), we obtain the following variational formulation of problem (6)-(17) in terms of displacement, damage and adhesion fields.

Problem 6. Find a displacement field $u:[0, T] \rightarrow V$, a damage field $\zeta:[0, T] \rightarrow H^{1}(\Omega)$ and a bonding field $\beta:[0, T] \rightarrow L^{\infty}\left(\Gamma_{3}\right)$ such that

$$
\begin{aligned}
& \langle\ddot{u}(t), w-\dot{u}(t)\rangle_{V^{*} \times V}+(\mathcal{A}(\varepsilon(\dot{u}(t))), \varepsilon(w-\dot{u}(t)))_{\mathcal{Q}} \\
& \quad+(\mathcal{B}(\varepsilon(u(t)), \zeta(t)), \varepsilon(w-\dot{u}(t)))_{\mathcal{Q}}+j_{\mathrm{ad}}(\beta(t), u(t), w-\dot{u}(t)) \\
& \quad+j_{\tau}(w)-j_{\tau}(\dot{u}(t)) \geq(f(t), w-\dot{u}(t))_{V}, \quad \forall w \in V, \text { a.e. } t \in(0, T), \\
& \zeta(t) \in \mathcal{K}, \\
& \quad(\dot{\zeta}(t), \psi-\zeta(t))_{L^{2}(\Omega)}+\kappa(\nabla \zeta(t), \nabla \psi-\nabla \zeta(t))_{H} \\
& \quad \geq(\mathcal{G}(\varepsilon(u(t)), \zeta(t)), \psi-\zeta(t))_{L^{2}(\Omega)}, \quad \forall \psi \in \mathcal{K}, \text { a.e. } t \in(0, T), \\
& \dot{\beta}(t)=H_{\mathrm{ad}}\left(\beta(t), \theta_{\beta}(t),\left|R_{\tau}\left(u_{\tau}(t)\right)\right|\right), \quad \text { a.e. } t \in(0, T), \\
& \beta(0)=\beta_{0}, \\
& \zeta(0)=\zeta_{0}, \\
& \text { (i) } u(0)=u_{0}, \quad \text { (ii) } \dot{u}(0)=v_{0} .
\end{aligned}
$$

4. Existence and uniqueness of a weak solution. The following theorem is the main result of this paper.

Theorem 7. Assume (21)-(31) are fulfilled. Then problem (46)-(51) has a unique solution $\{u, \beta, \zeta\}$. Moreover, the solution satisfies

$$
\begin{aligned}
& u \in W^{1,2}(0, T ; V) \cap C^{1}([0, T] ; H), \quad \ddot{u} \in L^{2}\left(0, T ; V^{*}\right), \\
& \beta \in W^{1, \infty}\left(0, T ; L^{\infty}\left(\Gamma_{3}\right)\right), \quad 0 \leq \beta(t) \leq 1, \text { a.e. } x \in \Gamma_{3}, \forall t \in[0, T], \\
& \zeta \in W^{1,2}\left(0, T ; L^{2}(\Omega)\right) \cap L^{2}\left(0, T ; H^{1}(\Omega)\right) .
\end{aligned}
$$

The proof will be carried out in several steps. In the rest of this paper, the same letter $c$ will be used to denote different positive constants independent of $t \in(0, T)$.

SteP 1. Consider the following problem.

Problem 8. Let $\eta \in L^{2}(0, T ; V)$. Find a function $\zeta_{\eta}:[0, T] \rightarrow H^{1}(\Omega)$ such that 
$(55)$

$$
\left\{\begin{array}{l}
\zeta_{\eta}(t) \in \mathcal{K}, \\
\left.\quad \dot{\zeta}_{\eta}(t), \psi-\zeta_{\eta}(t)\right)_{L^{2}(\Omega)}+\kappa\left(\nabla \zeta_{\eta}(t), \nabla \psi-\nabla \zeta_{\eta}(t)\right)_{H} \\
\quad \geq\left(\mathcal{G}\left(\varepsilon(\eta(t)), \zeta_{\eta}(t)\right), \psi-\zeta_{\eta}(t)\right)_{L^{2}(\Omega)}, \forall \psi \in \mathcal{K}, \text { a.e. } t \in(0, T),
\end{array}\right.
$$

(56) $\zeta_{\eta}(0)=\zeta_{0}$.

Lemma 9. Assume that (23) and (30) hold. Then problem (55)-(56) has a unique solution $\zeta_{\eta}$ which satisfies

$$
\zeta_{\eta} \in W^{1,2}\left(0, T ; L^{2}(\Omega)\right) \cap L^{2}\left(0, T ; H^{1}(\Omega)\right) .
$$

Furthermore, there is a constant $c>0$ such that for all $\eta_{1}, \eta_{2} \in L^{2}(0, T ; V)$,

$$
\left\|\zeta_{\eta_{1}}(t)-\zeta_{\eta_{2}}(t)\right\|_{L^{2}(\Omega)}^{2} \leq c \int_{0}^{t}\left\|\eta_{1}(s)-\eta_{2}(s)\right\|_{V}^{2} d s, \quad \forall t \in[0, T],
$$

where $\zeta_{\eta_{i}}(i=1,2)$ is the solution corresponding to $\eta_{i}$.

Proof. Let $(\eta, \phi) \in L^{2}(0, T ; V) \times L^{2}\left(0, T ; L^{2}(\Omega)\right)$. From 23 we can define $f_{\eta \phi} \in L^{2}\left(0, T ; L^{2}(\Omega)\right)$ by

$$
f_{\eta \phi}(t)=\mathcal{G}(\varepsilon(\eta(t)), \phi(t)), \quad \text { a.e. } t \in(0, T) .
$$

On the other hand, let $a: H^{1}(\Omega) \times H^{1}(\Omega) \rightarrow \mathbb{R}$ be the bilinear form defined by

$$
a(\varphi, \psi)=\kappa(\nabla \varphi, \nabla \psi)_{H}, \quad \forall \varphi, \psi \in H^{1}(\Omega) .
$$

After some algebraic computations, we find that $a$ satisfies (5) and it is continuous and symmetric on $H^{1}(\Omega)$. Therefore, applying Proposition 3 with $X=H^{1}(\Omega), Y=L^{2}(\Omega)$ and $K=\mathcal{K}$, we can see that, for each $(\eta, \phi) \in$ $L^{2}(0, T ; V) \times L^{2}\left(0, T ; L^{2}(\Omega)\right)$, the system

$$
\left\{\begin{array}{l}
\zeta_{\eta \phi}(t) \in \mathcal{K}, \\
\quad\left(\dot{\zeta}_{\eta \phi}(t), \psi-\zeta_{\eta \phi}(t)\right)_{L^{2}(\Omega)}+\kappa\left(\nabla \zeta_{\eta \phi}(t), \nabla \psi-\nabla \zeta_{\eta \phi}(t)\right)_{H} \\
\quad \geq\left(\mathcal{G}(\varepsilon(\eta(t)), \phi(t)), \psi-\zeta_{\eta \phi}(t)\right)_{L^{2}(\Omega)}, \quad \forall \psi \in \mathcal{K}, \text { a.e. } t \in(0, T),
\end{array}\right.
$$

(60) $\zeta_{\eta \phi}(0)=\zeta_{0}$

has a unique solution $\zeta_{\eta \phi}$ which satisfies

$$
\zeta_{\eta \phi} \in W^{1,2}\left(0, T ; L^{2}(\Omega)\right) \cap L^{2}\left(0, T ; H^{1}(\Omega)\right) .
$$

To continue, assume $\zeta_{\eta \phi_{1}}, \zeta_{\eta \phi_{2}}$ are two solutions to system $59-(60)$, corresponding to $(\eta, \phi)=\left(\eta, \phi_{1}\right)$ and $(\eta, \phi)=\left(\eta, \phi_{2}\right)$, respectively. Then 


$$
\begin{aligned}
& \int_{0}^{t}\left(\dot{\zeta}_{\eta \phi_{1}}(s)-\dot{\zeta}_{\eta \phi_{2}}(s), \zeta_{\eta \phi_{1}}(s)-\zeta_{\eta \phi_{2}}(s)\right)_{L^{2}(\Omega)} d s \\
&+\kappa \int_{0}^{t} a\left(\zeta_{\eta \phi_{1}}(s)-\zeta_{\eta \phi_{2}}(s), \zeta_{\eta \phi_{1}}(s)-\zeta_{\eta \phi_{2}}(s)\right) d s \\
& \leq \int_{0}^{t}\left(\mathcal{G}\left(\varepsilon(\eta(s)), \phi_{1}(s)\right)-\mathcal{G}\left(\varepsilon(\eta(s)), \phi_{2}(s)\right), \zeta_{\eta \phi_{1}}(s)-\zeta_{\eta \phi_{2}}(s)\right)_{L^{2}(\Omega)} d s
\end{aligned}
$$

and using the inequality

$$
\lambda \delta \leq \frac{1}{2} \lambda^{2}+\frac{1}{2} \delta^{2}, \quad \forall \lambda, \delta \in \mathbb{R},
$$

we deduce that

$$
\begin{aligned}
\left\|\zeta_{\eta \phi_{1}}(t)-\zeta_{\eta \phi_{2}}(t)\right\|_{L^{2}(\Omega)}^{2} \leq & c \int_{0}^{t}\left\|\phi_{1}(s)-\phi_{2}(s)\right\|_{L^{2}(\Omega)}^{2} d s \\
& +c \int_{0}^{t}\left\|\zeta_{\eta \phi_{1}}(s)-\zeta_{\eta \phi_{2}}(s)\right\|_{L^{2}(\Omega)}^{2} d s .
\end{aligned}
$$

From Gronwall's inequality, we obtain

$$
\left\|\zeta_{\eta \phi_{1}}(t)-\zeta_{\eta \phi_{2}}(t)\right\|_{L^{2}(\Omega)}^{2} \leq c \int_{0}^{t}\left\|\phi_{1}(s)-\phi_{2}(s)\right\|_{L^{2}(\Omega)}^{2} d s, \quad \forall t \in[0, T] .
$$

Now, for each $\eta \in L^{2}(0, T ; V)$, consider the operator $\Phi_{\eta}: L^{2}\left(0, T ; L^{2}(\Omega)\right) \rightarrow$ $L^{2}\left(0, T ; L^{2}(\Omega)\right)$ defined by

$$
\Phi_{\eta} \phi=\zeta_{\eta \phi}, \quad \forall \phi \in L^{2}\left(0, T ; L^{2}(\Omega)\right),
$$

where $\zeta_{\eta \phi}$ is the unique solution satisfying $\left.(59)-60\right)$. Using (62)-63) we get

$$
\left\|\Phi_{\eta} \phi_{1}(t)-\Phi_{\eta} \phi_{2}(t)\right\|_{L^{2}(\Omega)}^{2} \leq c \int_{0}^{t}\left\|\phi_{1}(s)-\phi_{2}(s)\right\|_{L^{2}(\Omega)}^{2} d s, \quad \forall t \in[0, T] .
$$

Reiterating the last inequality $n$ times, we infer that

$$
\left\|\Phi_{\eta}^{n} \phi_{1}-\Phi_{\eta}^{n} \phi_{2}\right\|_{L^{2}\left(0, T ; L^{2}(\Omega)\right)}^{2} \leq \frac{(c T)^{n}}{n !}\left\|\phi_{1}-\phi_{2}\right\|_{L^{2}\left(0, T ; L^{2}(\Omega)\right)}^{2},
$$

which implies that, for $n$ sufficiently large, a power $\Phi_{\eta}^{n}$ of $\Phi_{\eta}$ is a contraction in the Banach space $L^{2}\left(0, T ; L^{2}(\Omega)\right)$. Therefore, $\Phi_{\eta}$ has a unique fixed point $\phi_{\eta}^{*} \in L^{2}\left(0, T ; L^{2}(\Omega)\right)$. Now, let $\eta \in L^{2}(0, T ; V)$, let $\phi_{\eta}^{*}$ be the fixed point of $\Phi_{\eta}$, let $\zeta_{\eta}=\zeta_{\eta \phi_{\eta}^{*}}$ and keeping in mind $(59)-(61)$, it is straightforward to see that $\zeta_{\eta}$ is a unique solution to problem (55)-(56) such that (57) holds. Finally, assume $\zeta_{\eta_{1}}, \zeta_{\eta_{2}}$ are two solutions to problem (55)-(56), corresponding to $\eta_{1}$ 
and $\eta_{2}$, respectively. Then we get

$$
\begin{aligned}
\| \zeta_{\eta_{1}}(t) & -\zeta_{\eta_{2}}(t) \|_{L^{2}(\Omega)}^{2} \\
& \leq c \int_{0}^{t}\left\|\eta_{1}(s)-\eta_{2}(s)\right\|_{V}^{2} d s+c \int_{0}^{t}\left\|\zeta_{\eta_{1}}(s)-\zeta_{\eta_{2}}(s)\right\|_{L^{2}(\Omega)}^{2} d s, \quad \forall t \in[0, T] .
\end{aligned}
$$

Thus, using Gronwall's inequality, we obtain (58).

Now, consider the following problem.

Problem 10. Let $\eta \in L^{2}(0, T ; V)$. Find a function $\beta_{\eta}:[0, T] \rightarrow L^{\infty}\left(\Gamma_{3}\right)$ such that

$$
\begin{aligned}
& \dot{\beta}_{\eta}(t)=H_{\mathrm{ad}}\left(\beta_{\eta}(t), \theta_{\beta_{\eta}}(t),\left|R_{\tau}\left(\eta_{\tau}(t)\right)\right|\right), \quad \text { a.e. } t \in(0, T), \\
& \beta_{\eta}(0)=\beta_{0},
\end{aligned}
$$

where

$$
\theta_{\beta_{\eta}}(t)=\int_{0}^{t} \beta_{\eta}(s) d s, \quad \forall t \in[0, T] .
$$

Lemma 11. Assume (25) and (29) are fulfilled. Then problem (64)-66) has a unique solution which satisfies

$$
\left\{\begin{array}{l}
\text { (i) } \beta_{\eta} \in W^{1, \infty}\left(0, T ; L^{\infty}\left(\Gamma_{3}\right)\right), \\
\text { (ii) } 0 \leq \beta_{\eta}(t) \leq 1, \forall t \in[0, T] \text {, a.e. } x \in \Gamma_{3} .
\end{array}\right.
$$

Moreover, there exists a constant $c>0$ such that for all $\eta_{1}, \eta_{2} \in L^{2}(0, T ; V)$,

$$
\left\|\beta_{\eta_{1}}(t)-\beta_{\eta_{2}}(t)\right\|_{L^{2}\left(\Gamma_{3}\right)}^{2} \leq c \int_{0}^{t}\left\|\eta_{1}(s)-\eta_{2}(s)\right\|_{V}^{2} d s, \quad \forall t \in[0, T],
$$

where $\beta_{\eta_{i}}(i=1,2)$ is the solution corresponding to $\eta_{i}$.

Proof. Fix $(\eta, \theta) \in L^{2}(0, T ; V) \times L^{\infty}\left(0, T ; L^{\infty}\left(\Gamma_{3}\right)\right)$ and consider $F$ : $[0, T] \times L^{\infty}\left(\Gamma_{3}\right) \rightarrow L^{\infty}\left(\Gamma_{3}\right)$ defined by

$$
F(t, \beta)=H_{\text {ad }}\left(\beta, \theta(t),\left|R_{\tau}\left(\eta_{\tau}(t)\right)\right|\right), \quad \forall \beta \in L^{\infty}\left(\Gamma_{3}\right) \text {, a.e. } t \in(0, T) .
$$

Let $\beta_{1}, \beta_{2} \in L^{\infty}\left(\Gamma_{3}\right)$. From $(69)$ and $(25)$, we obtain

$$
\begin{aligned}
\mid F\left(t, \beta_{1}\right)- & F\left(t, \beta_{2}\right) \mid \\
& \leq\left|H_{\text {ad }}\left(\beta_{1}, \theta(t),\left|R_{\tau}\left(\eta_{\tau}(t)\right)\right|\right)-H_{\mathrm{ad}}\left(\beta_{2}, \theta(t),\left|R_{\tau}\left(\eta_{\tau}(t)\right)\right|\right)\right| \\
& \leq L_{H_{\mathrm{ad}}}\left|\beta_{1}-\beta_{2}\right|, \quad \text { a.e. } t \in(0, T),
\end{aligned}
$$

which implies that

$$
\left\|F\left(t, \beta_{1}\right)-F\left(t, \beta_{2}\right)\right\|_{L^{\infty}\left(\Gamma_{3}\right)} \leq L_{H_{\text {ad }}}\left\|\beta_{1}-\beta_{2}\right\|_{L^{\infty}\left(\Gamma_{3}\right)}, \quad \text { a.e. } t \in(0, T) .
$$

Hence, $F$ is Lipschitz continuous with respect to the second argument, uniformly in time. Moreover, $t \mapsto F(t, \beta)$ belongs to $L^{\infty}\left(0, T ; L^{\infty}\left(\Gamma_{3}\right)\right)$, 
$\forall \beta \in L^{\infty}\left(\Gamma_{3}\right)$. Thus, using Proposition 1 , we deduce that there exists a unique $\beta_{\eta \theta} \in W^{1, \infty}\left(0, T ; L^{\infty}\left(\Gamma_{3}\right)\right)$ such that

$$
\begin{aligned}
& \dot{\beta}_{\eta \theta}(t)=H_{\mathrm{ad}}\left(\beta_{\eta \theta}(t), \theta(t),\left|R_{\tau}\left(\eta_{\tau}(t)\right)\right|\right), \quad \text { a.e. } t \in(0, T), \\
& \beta_{\eta \theta}(0)=\beta_{0} .
\end{aligned}
$$

Moreover, from (70)-21), 25), 29) and using arguments similar to those in [16], 22], we deduce that

$$
0 \leq \beta_{\eta \theta}(t) \leq 1, \quad \forall t \in[0, T], \quad \text { a.e. } x \in \Gamma_{3} .
$$

Now, let $\beta_{\eta \theta_{1}}, \beta_{\eta \theta_{2}}$ be two solutions satisfying (70)-21). It follows from (25) that

$$
\begin{aligned}
\left\|\beta_{\eta \theta_{1}}(t)-\beta_{\eta \theta_{2}}(t)\right\|_{L^{\infty}\left(\Gamma_{3}\right)} \leq & c \int_{0}^{t}\left\|\beta_{\eta \theta_{1}}(s)-\beta_{\eta \theta_{2}}(s)\right\|_{L^{\infty}\left(\Gamma_{3}\right)} d s \\
& +c \int_{0}^{t}\left\|\theta_{1}(s)-\theta_{2}(s)\right\|_{L^{\infty}\left(\Gamma_{3}\right)} d s .
\end{aligned}
$$

Using Gronwall's inequality we get

$$
\left\|\beta_{\eta \theta_{1}}(t)-\beta_{\eta \theta_{2}}(t)\right\|_{L^{\infty}\left(\Gamma_{3}\right)} \leq c \int_{0}^{t}\left\|\theta_{1}(s)-\theta_{2}(s)\right\|_{L^{\infty}\left(\Gamma_{3}\right)} d s .
$$

To continue, for each $\eta \in L^{2}(0, T ; V)$, consider the operator $\Theta_{\eta}$ : $L^{\infty}\left(0, T ; L^{\infty}\left(\Gamma_{3}\right)\right) \rightarrow L^{\infty}\left(0, T ; L^{\infty}\left(\Gamma_{3}\right)\right)$ defined by

$$
\Theta_{\eta} \theta(t)=\int_{0}^{t} \beta_{\eta \theta}(s) d s, \quad \forall t \in[0, T], \forall \theta \in L^{\infty}\left(0, T ; L^{\infty}\left(\Gamma_{3}\right)\right),
$$

where $\beta_{\eta \theta}$ is the unique solution of $(70--71)$. Using $(73)-(74)$ we have

$$
\begin{aligned}
\left\|\Theta_{\eta} \theta_{1}(t)-\Theta_{\eta} \theta_{2}(t)\right\|_{L^{\infty}\left(\Gamma_{3}\right)} & \leq \int_{0}^{t}\left\|\beta_{\eta \theta_{1}}(s)-\beta_{\eta \theta_{2}}(s)\right\|_{L^{\infty}\left(\Gamma_{3}\right)} d s \\
& \leq c \int_{00}^{t} \int_{0}^{s}\left\|\theta_{1}(r)-\theta_{2}(r)\right\|_{L^{\infty}\left(\Gamma_{3}\right)} d r d s \\
& \leq c \int_{0}^{t}\left\|\theta_{1}(r)-\theta_{2}(r)\right\|_{L^{\infty}\left(\Gamma_{3}\right)} d r, \quad \forall t \in[0, T] .
\end{aligned}
$$

Reiterating this inequality $n$ times, we obtain

$$
\left\|\Theta_{\eta} \theta_{1}-\Theta_{\eta} \theta_{2}\right\|_{L^{\infty}\left(0, T ; L^{\infty}\left(\Gamma_{3}\right)\right)} \leq \frac{(c T)^{n}}{n !}\left\|\theta_{1}-\theta_{2}\right\|_{L^{\infty}\left(0, T ; L^{\infty}\left(\Gamma_{3}\right)\right)},
$$

which implies that, for $n$ sufficiently large, a power $\Theta_{\eta}^{n}$ of $\Theta_{\eta}$ is a contraction in the Banach space $L^{\infty}\left(0, T ; L^{\infty}\left(\Gamma_{3}\right)\right)$. Therefore, $\Theta_{\eta}$ has a unique fixed 
point $\theta_{\eta}^{*} \in L^{\infty}\left(0, T ; L^{\infty}\left(\Gamma_{3}\right)\right)$ given by

$$
\theta_{\eta}^{*}(t)=\int_{0}^{t} \beta_{\eta \theta_{\eta}^{*}}(s) d s, \quad \forall t \in[0, T] .
$$

Now, let $\eta \in L^{2}(0, T ; V)$, let $\theta_{\eta}^{*}$ be the fixed point of $\Theta_{\eta}$, let $\beta_{\eta}=\beta_{\eta \theta_{\eta}^{*}}$, let $\theta_{\beta_{\eta}}=\theta_{\eta}^{*}$ and keeping in mind $(70)-(72)$, it is straightforward to see that $\beta_{\eta}$ is a unique solution to problem (64)-(66) such that (67) holds.

Finally, assume $\beta_{\eta_{1}}, \beta_{\eta_{2}}$ are two solutions to problem $(64)-(66)$, corresponding to $\eta_{1}$ and $\eta_{2}$, respectively. Then from (25) and 18$)$ we obtain

$$
\begin{aligned}
& \left\|\beta_{\eta_{1}}(t)-\beta_{\eta_{2}}(t)\right\|_{L^{2}\left(\Gamma_{3}\right)}^{2} \\
& \leq c \int_{0}^{t}\left\|\beta_{\eta_{1}}(s)-\beta_{\eta_{2}}(s)\right\|_{L^{2}\left(\Gamma_{3}\right)}^{2} d s+c \int_{0}^{t}\left\|\theta_{\eta_{1}}^{*}(s)-\theta_{\eta_{2}}^{*}(s)\right\|_{L^{2}\left(\Gamma_{3}\right)}^{2} d s \\
& \quad+c \int_{0}^{t}\left\|\eta_{1}(s)-\eta_{2}(s)\right\|_{L^{2}\left(\Gamma_{3} ; \mathbb{R}^{d}\right)}^{2} d s \\
& \leq c \int_{0}^{t}\left\|\beta_{\eta_{1}}(s)-\beta_{\eta_{2}}(s)\right\|_{L^{2}\left(\Gamma_{3}\right)}^{2} d s+c \int_{0}^{t} \int_{0}^{t}\left\|\beta_{\eta_{1}}(r)-\beta_{\eta_{2}}(r)\right\|_{L^{2}\left(\Gamma_{3}\right)}^{2} d r d s \\
& \quad+c \int_{0}^{t}\left\|\eta_{1}(s)-\eta_{2}(s)\right\|_{L^{2}\left(\Gamma_{3} ; \mathbb{R}^{d}\right)}^{2} d s,
\end{aligned}
$$

which leads to

$$
\begin{aligned}
\left\|\beta_{\eta_{1}}(t)-\beta_{\eta_{2}}(t)\right\|_{L^{2}\left(\Gamma_{3}\right)}^{2} \leq & c \int_{0}^{t}\left\|\beta_{\eta_{1}}(r)-\beta_{\eta_{2}}(r)\right\|_{L^{2}\left(\Gamma_{3}\right)}^{2} d r \\
& +c \int_{0}^{t}\left\|\eta_{1}(s)-\eta_{2}(s)\right\|_{L^{2}\left(\Gamma_{3}, \mathbb{R}^{d}\right)}^{2} d s,
\end{aligned}
$$

which, together with Gronwall's inequality (20), gives (68).

STEP 2. Let $\alpha>0$. We define a regularized functional $j_{\alpha}: V \rightarrow \mathbb{R}$ by

$$
j_{\alpha}(w)=\int_{\Gamma_{3}} g_{b} \sqrt{\left|w_{\tau}\right|^{2}+\alpha^{2}} d a, \quad \forall w \in V,
$$

which represents an approximation of $j_{\tau}$. More precisely,

$$
\left|j_{\alpha}(w)-j_{\tau}(w)\right| \leq \alpha\left\|g_{b}\right\|_{L^{1}\left(\Gamma_{3}\right)}, \quad \forall w \in V .
$$

Moreover, $j_{\alpha}$ has a Gâteaux derivative $j_{\alpha}^{\prime}: V \rightarrow V^{*}$ given by

$$
\left\langle j_{\alpha}^{\prime}(y), w\right\rangle_{V^{*} \times V}=\int_{\Gamma_{3}} \frac{g_{b}}{\sqrt{\left|y_{\tau}\right|^{2}+\alpha^{2}}} y_{\tau} \cdot w_{\tau} d a, \quad \forall w, y \in V .
$$


Since $j_{\alpha}$ is a convex function, it follows (see, e.g., [19]) that $j_{\alpha}^{\prime}$ is a hemicontinuous operator and satisfies

$$
\left\langle j_{\alpha}^{\prime}(y), w-y\right\rangle_{V^{*} \times V} \leq j_{\alpha}(w)-j_{\alpha}(y), \quad \forall w, y \in V,
$$

which leads to

$$
\left\langle j_{\alpha}^{\prime}(y)-j_{\alpha}^{\prime}(w), y-w\right\rangle_{V^{*} \times V} \geq 0, \quad \forall w, y \in V .
$$

To continue, let $\alpha>0$, let $\eta \in L^{2}(0, T ; V)$, let $\beta_{\eta}$ be the unique solution of problem (64)-(66), let $\zeta_{\eta}$ be the unique solution of problem (55)-(56). We can define $f_{\eta} \in L^{2}\left(0, T ; V^{*}\right)$ by

$$
\begin{aligned}
\left\langle f_{\eta}(t), w\right\rangle_{V^{*} \times V}= & (f(t), w)_{V}-\left(\mathcal{B}\left(\varepsilon(\eta(t)), \zeta_{\eta}(t)\right), \varepsilon(w)\right)_{\mathcal{Q}} \\
& -j_{\text {ad }}\left(\beta_{\eta}(t), \eta(t), w\right), \quad \text { a.e. } t \in(0, T), \forall w \in V,
\end{aligned}
$$

and we consider the following regularized problem.

Problem 12. Let $\alpha>0$ and let $\eta \in L^{2}(0, T ; V)$. Find a function $v_{\eta}^{\alpha}$ : $[0, T] \rightarrow V$ such that

$$
\begin{aligned}
& \left\langle\dot{v}_{\eta}^{\alpha}(t), w\right\rangle_{V^{*} \times V}+\left(\mathcal{A}\left(\varepsilon\left(v_{\eta}^{\alpha}(t)\right)\right), \varepsilon(w)\right)_{\mathcal{Q}}+\left\langle j_{\alpha}^{\prime}\left(v_{\eta}^{\alpha}(t)\right), w\right\rangle_{V^{*} \times V} \\
& =\left\langle f_{\eta}(t), w\right\rangle_{V^{*} \times V}, \quad \forall w \in V \text {, a.e. } t \in(0, T), \\
& v_{\eta}^{\alpha}(0)=v_{0}
\end{aligned}
$$

Lemma 13. Assume that 21)-(30) and (31)(ii) hold. Then problem (80)-81) has a unique solution $v_{\eta}^{\alpha}$ which satisfies

$$
v_{\eta}^{\alpha} \in L^{2}(0, T ; V) \cap C([0, T] ; H), \quad \dot{v}_{\eta}^{\alpha} \in L^{2}\left(0, T ; V^{*}\right) .
$$

Proof. Let $A: V \rightarrow V^{*}$ be defined by

$$
\langle A w, z\rangle_{V^{*} \times V}=(\mathcal{A}(\varepsilon(w)), \varepsilon(z))_{\mathcal{Q}}+\left\langle j_{\alpha}^{\prime}(w), z\right\rangle_{V^{*} \times V}, \quad \forall w, z \in V .
$$

From (21) and (78), we deduce that $A$ is a monotone operator. Moreover, using (83), 21] one has, for all $\lambda, \lambda_{0} \in \mathbb{R}$,

$$
\begin{aligned}
\mid\langle A(w+\lambda v)- & \left.A\left(w+\lambda_{0} v\right), z\right\rangle_{V^{*} \times V}\left|\leq L_{\mathcal{A}}\right| \lambda-\lambda_{0} \mid\|v\|_{V}\|z\|_{V} \\
& +\left|\left\langle j_{\alpha}^{\prime}(w+\lambda v)-j_{\alpha}^{\prime}\left(w+\lambda_{0} v\right), z\right\rangle_{V^{*} \times V}\right|, \quad \forall w, v, z \in V,
\end{aligned}
$$

and since $j_{\alpha}^{\prime}$ is hemicontinuous, it follows that $A$ is hemicontinuous from $V$ to $V^{*}$. To continue, since $\left\langle j_{\alpha}^{\prime}(v), v\right\rangle_{V^{*} \times V} \geq 0$, using (21) and (83) we get

$$
\begin{aligned}
m_{\mathcal{A}}\|v\|_{V}^{2} & \leq(\mathcal{A}(\varepsilon(v))-\mathcal{A}(\varepsilon(0)), \varepsilon(v)-\varepsilon(0))_{\mathcal{Q}} \\
& \leq(\mathcal{A}(\varepsilon(v)), \varepsilon(v))_{\mathcal{Q}}-(\mathcal{A}(\varepsilon(0)), \varepsilon(v))_{\mathcal{Q}} \\
& \leq(\mathcal{A}(\varepsilon(v)), \varepsilon(v))_{\mathcal{Q}}+\left\langle j_{\alpha}^{\prime}(v), v\right\rangle_{V^{*} \times V}-(\mathcal{A}(0), \varepsilon(v))_{\mathcal{Q}} \\
& \leq\langle A v, v\rangle_{V^{*} \times V}+\|\mathcal{A}(0)\|_{\mathcal{Q}}\|v\|_{V},
\end{aligned}
$$

which leads to

$$
m_{\mathcal{A}}\|v\|_{V}^{2} \leq\langle A v, v\rangle_{V^{*} \times V}+\frac{1}{2 m_{\mathcal{A}}}\|\mathcal{A}(0)\|_{\mathcal{Q}}^{2}+\frac{m_{\mathcal{A}}}{2}\|v\|_{V}^{2},
$$


which implies that

$$
\frac{m_{\mathcal{A}}}{2}\|v\|_{V}^{2}-\frac{1}{2 m_{\mathcal{A}}}\|\mathcal{A}(0)\|_{\mathcal{Q}}^{2} \leq\langle A v, v\rangle_{V^{*} \times V} .
$$

On the other hand, from (83), 20), 21) and (76), one has

$$
\begin{aligned}
\mid\langle A v & , w\rangle_{V^{*} \times V} \mid \\
& \leq\left|(\mathcal{A}(\varepsilon(v)), \varepsilon(w))_{\mathcal{Q}}\right|+\left|\left\langle j_{\alpha}^{\prime}(v), w\right\rangle_{V^{*} \times V}\right| \\
& \leq\left|(\mathcal{A}(\varepsilon(v))-\mathcal{A}(\varepsilon(0)), \varepsilon(w))_{\mathcal{Q}}\right|+\left|(\mathcal{A}(\varepsilon(0)), \varepsilon(w))_{\mathcal{Q}}\right|+\left|\left\langle j_{\alpha}^{\prime}(v), w\right\rangle_{V^{*} \times V}\right| \\
& \leq L_{\mathcal{A}}\|v\|_{V}\|w\|_{V}+\|\mathcal{A}(0)\|_{\mathcal{Q}}\|w\|_{V}+c_{0}\left\|g_{b}\right\|_{L^{2}\left(\Gamma_{3}\right)}\|w\|_{V},
\end{aligned}
$$

which implies that

$$
\|A v\|_{V^{*}} \leq L_{\mathcal{A}}\|v\|_{V}+\|\mathcal{A}(0)\|_{\mathcal{Q}}+c_{0}\left\|g_{b}\right\|_{L^{2}\left(\Gamma_{3}\right)} .
$$

Thus, $A$ satisfies conditions $(3)-44$ with $\mathcal{C}_{1}=m_{\mathcal{A}} / 2, \mathcal{C}_{2}=-\frac{1}{2 m_{\mathcal{A}}}\|\mathcal{A}(0)\|_{\mathcal{Q}}^{2}$ and $\mathcal{C}_{3}=\max \left(L_{\mathcal{A}},\|\mathcal{A}(0)\|_{\mathcal{Q}}+c_{0}\left\|g_{b}\right\|_{L^{2}\left(\Gamma_{3}\right)}\right)$. Now, keeping in mind (31)(ii), it follows from Proposition 2.2 that there exists a unique function $v_{\eta}^{\alpha}$ satisfying

$$
\begin{aligned}
& \dot{v}_{\eta}^{\alpha}(t)+A v_{\eta}^{\alpha}(t)=f_{\eta}(t) \quad \text { in } V^{*}, \text { a.e. } t \in(0, T), \\
& v_{\eta}^{\alpha}(0)=v_{0},
\end{aligned}
$$

such that 82 holds. Thus problem (80)-(81) has a unique solution $v_{\eta}^{\alpha}$ which satisfies 82 .

Step 3. Next, we introduce the space $\mathcal{W}=\left\{v \in \mathcal{V} ; \dot{v} \in \mathcal{V}^{*}\right\}$ which is a separable and reflexive Banach space equipped with the norm

$$
\|v\|_{\mathcal{W}}=\|v\|_{\mathcal{V}}+\|\dot{v}\|_{\mathcal{V}^{*}}
$$

where $\mathcal{V}=L^{2}(0, T ; V)$ and $\mathcal{V}^{*}=L^{2}\left(0, T ; V^{*}\right)$. We have the following result.

Lemma 14. There exists a function $v_{\eta} \in \mathcal{W}$ and a subsequence of $\left\{v_{\eta}^{\alpha}\right\}$, again denoted by $\left\{v_{\eta}^{\alpha}\right\}$, such that as $\alpha \rightarrow 0$, the following convergences hold:

$$
\begin{array}{cl}
v_{\eta}^{\alpha} \rightarrow v_{\eta} & \text { weakly in } L^{2}(0, T ; V), \\
\dot{v}_{\eta}^{\alpha} \rightarrow \dot{v}_{\eta} & \text { weakly in } L^{2}\left(0, T ; V^{*}\right), \\
v_{\eta}^{\alpha}(t) \rightarrow v_{\eta}(t) & \text { weakly in } H, \forall t \in[0, T] .
\end{array}
$$

Proof. It follows from that

$$
\begin{array}{r}
\left\langle\dot{v}_{\eta}^{\alpha}(s), v_{\eta}^{\alpha}(s)\right\rangle_{V^{*} \times V}+\left(\mathcal{A}\left(\varepsilon\left(v_{\eta}^{\alpha}(s)\right)\right), \varepsilon\left(v_{\eta}^{\alpha}(s)\right)\right)_{\mathcal{Q}}+\left\langle j_{\alpha}^{\prime}\left(v_{\eta}^{\alpha}(s)\right), v_{\eta}^{\alpha}(s)\right\rangle_{V^{*} \times V} \\
=\left\langle f_{\eta}(s), v_{\eta}^{\alpha}(s)\right\rangle_{V^{*} \times V}, \quad s \in(0, T),
\end{array}
$$

and since $\left\langle j_{\alpha}^{\prime}\left(v_{\eta}^{\alpha}(s)\right), v_{\eta}^{\alpha}(s)\right\rangle_{V^{*} \times V} \geq 0$, we get

$$
\begin{aligned}
&\left\langle\dot{v}_{\eta}^{\alpha}(s), v_{\eta}^{\alpha}(s)\right\rangle_{V^{*} \times V}+\left(\mathcal{A}\left(\varepsilon\left(v_{\eta}^{\alpha}(s)\right)\right)-\mathcal{A}(\varepsilon(0)), \varepsilon\left(v_{\eta}^{\alpha}(s)\right)\right)_{\mathcal{Q}} \\
& \leq\left\langle f_{\eta}(s), v_{\eta}^{\alpha}(s)\right\rangle_{V^{*} \times V}-\left(\mathcal{A}(\varepsilon(0)), \varepsilon\left(v_{\eta}^{\alpha}(s)\right)\right)_{\mathcal{Q}}, \quad s \in(0, T),
\end{aligned}
$$


and using (21), we deduce that

$$
\begin{aligned}
\left\langle\dot{v}_{\eta}^{\alpha}(s), v_{\eta}^{\alpha}(s)\right\rangle_{V^{*} \times V}+m_{\mathcal{A}}\left\|v_{\eta}^{\alpha}(s)\right\|_{V}^{2} \leq & \frac{1}{m_{\mathcal{A}}}\left\|f_{\eta}(s)\right\|_{V^{*}}^{2}+\frac{m_{\mathcal{A}}}{4}\left\|v_{\eta}^{\alpha}(s)\right\|_{V}^{2} \\
& +\frac{1}{m_{\mathcal{A}}}\|\mathcal{A}(0)\|_{\mathcal{Q}}^{2}+\frac{m_{\mathcal{A}}}{4}\left\|v_{\eta}^{\alpha}(s)\right\|_{V}^{2} .
\end{aligned}
$$

Integrating both sides, one has

$$
\left\|v_{\eta}^{\alpha}(t)\right\|_{H}^{2}+\int_{0}^{t}\left\|v_{\eta}^{\alpha}(s)\right\|_{V}^{2} d s \leq c \int_{0}^{t}\left\|f_{\eta}(s)\right\|_{V^{*}}^{2} d s+\left\|v_{0}\right\|_{H}^{2}+c, \quad \forall t \in[0, T] .
$$

From this, we get

$$
\left\|v_{\eta}^{\alpha}\right\|_{L^{\infty}(0, T ; H)}+\left\|v_{\eta}^{\alpha}\right\|_{L^{2}(0, T ; V)} \leq c .
$$

To continue, using (84)-85), we obtain

$\left\|\dot{v}_{\eta}^{\alpha}(s)\right\|_{V^{*}} \leq L_{\mathcal{A}}\left\|v_{\eta}^{\alpha}(s)\right\|_{V}+\|\mathcal{A}(0)\|_{\mathcal{Q}}+c_{0}\left\|g_{b}\right\|_{L^{2}\left(\Gamma_{3}\right)}+\left\|f_{\eta}(s)\right\|_{V^{*}}, s \in(0, T)$, which gives

$$
\left\|\dot{v}_{\eta}^{\alpha}(s)\right\|_{V^{*}}^{2} \leq c\left\|v_{\eta}^{\alpha}(s)\right\|_{V}^{2}+c\left\|f_{\eta}(s)\right\|_{V^{*}}^{2}+c, \quad \text { a.e. } s \in(0, T) ;
$$

integrating both sides on $(0, t)$, we get

$$
\int_{0}^{t}\left\|\dot{v}_{\eta}^{\alpha}(s)\right\|_{V^{*}}^{2} d s \leq c \int_{0}^{t}\left\|v_{\eta}^{\alpha}(s)\right\|_{V}^{2} d s+c \int_{0}^{t}\left\|f_{\eta}(s)\right\|_{V^{*}}^{2} d s+c,
$$

which, together with 90 , implies that

$$
\left\|\dot{v}_{\eta}^{\alpha}\right\|_{L^{2}\left(0, T ; V^{*}\right)} \leq c .
$$

In (90- (91), $c$ is a positive constant independent of $\alpha$. Thus, from standard compactness arguments, there exists a function $v_{\eta} \in L^{2}(0, T ; V) \cap$ $W^{1,2}\left(0, T ; V^{*}\right)$ and a subsequence of $\left\{v_{\eta}^{\alpha}\right\}$, still denoted by $\left\{v_{\eta}^{\alpha}\right\}$, such that the convergences (87)- 88$)$ hold. Now, since the inclusion map $\mathcal{W} \subset C([0, T] ; H)$ is continuous, the convergence 89 follows from $87-88$.

Lemma 15. For any $z \in L^{2}(0, T ; V)$, the following properties hold:

$$
\begin{aligned}
\liminf _{\alpha \rightarrow 0} \int_{0}^{T}\left\langle\dot{v}_{\eta}^{\alpha}(s), v_{\eta}^{\alpha}(s)-z(s)\right\rangle_{V^{*} \times V} d s & \geq \int_{0}^{T}\left\langle\dot{v}_{\eta}(s), v_{\eta}(s)-z(s)\right\rangle_{V^{*} \times V} d s, \\
\liminf _{\alpha \rightarrow 0} \int_{0}^{T}\left[j_{\alpha}\left(v_{\eta}^{\alpha}(s)\right)-j_{\alpha}(z(s))\right] d s & \geq \int_{0}^{T}\left[j_{\tau}\left(v_{\eta}(s)\right)-j_{\tau}(z(s))\right] d s,
\end{aligned}
$$




$$
\begin{aligned}
\liminf _{\alpha \rightarrow 0} \int_{0}^{T}\left(\mathcal{A}\left(\varepsilon\left(v_{\eta}^{\alpha}(s)\right)\right), \varepsilon\left(v_{\eta}^{\alpha}(s)-z(s)\right)\right)_{\mathcal{Q}} d s & \\
& \geq \int_{0}^{T}\left(\mathcal{A}\left(\varepsilon\left(v_{\eta}(s)\right)\right), \varepsilon\left(v_{\eta}(s)-z(s)\right)\right)_{\mathcal{Q}} d s .
\end{aligned}
$$
obtain

Proof. Using $(89)$ and $(81)$ we find that $v_{\eta}^{\alpha}(0)=v_{\eta}(0)$. Moreover, we

$$
\begin{array}{r}
\liminf _{\alpha \rightarrow 0} \int_{0}^{T}\left\langle\dot{v}_{\eta}^{\alpha}(s), v_{\eta}^{\alpha}(s)\right\rangle_{V^{*} \times V} d s=\liminf _{\alpha \rightarrow 0}\left(\frac{1}{2}\left\|v_{\eta}^{\alpha}(T)\right\|_{H}^{2}-\frac{1}{2}\left\|v_{\eta}(0)\right\|_{H}^{2}\right) \\
\geq \frac{1}{2}\left\|v_{\eta}(T)\right\|_{H}^{2}-\frac{1}{2}\left\|v_{\eta}(0)\right\|_{H}^{2} \geq \int_{0}^{T}\left\langle\dot{v}_{\eta}(s), v_{\eta}(s)\right\rangle_{V^{*} \times V} d s,
\end{array}
$$

which together with (88) implies (92).

To establish (93), we write

$$
\begin{aligned}
\int_{0}^{T}\left[j_{\alpha}\left(v_{\eta}^{\alpha}(s)\right)-\right. & \left.j_{\alpha}(z(s))\right] d s \\
= & \int_{0}^{T}\left[j_{\alpha}\left(v_{\eta}^{\alpha}(s)\right)-j_{\tau}\left(v_{\eta}^{\alpha}(s)\right)\right] d s+\int_{0}^{T}\left[j_{\tau}(z(s))-j_{\alpha}(z(s))\right] d s \\
& +\int_{0}^{T}\left[j_{\tau}\left(v_{\eta}^{\alpha}(s)\right)-j_{\tau}(z(s))\right] d s .
\end{aligned}
$$

Keeping in mind (75), (87) and using standard lower semicontinuity arguments, in the last equality, we get

$$
\begin{aligned}
\liminf _{\alpha \rightarrow 0} \int_{0}^{T}\left[j_{\alpha}\left(v_{\eta}^{\alpha}(s)\right)-j_{\alpha}(z(s))\right] d s & =\liminf _{\alpha \rightarrow 0} \int_{0}^{T}\left[j_{\tau}\left(v_{\eta}^{\alpha}(s)\right)-j_{\tau}(z(s))\right] d s \\
& \geq \int_{0}^{T}\left[j_{\tau}\left(v_{\eta}(s)\right)-j_{\tau}(z(s))\right] d s .
\end{aligned}
$$

To continue, let $\mathcal{F}: \mathcal{V} \rightarrow \mathcal{V}^{*}$ be the operator defined by

$$
\langle\mathcal{F} w, z\rangle_{\mathcal{V}^{*} \times \mathcal{V}}=\int_{0}^{T}(\mathcal{A}(\varepsilon(w(s))), \varepsilon(z(s)))_{\mathcal{Q}} d s, \quad \forall w, z \in \mathcal{V} .
$$

From (80), (77) and (95), we get

$$
\begin{aligned}
&\left\langle\mathcal{F} v_{\eta}^{\alpha}, v_{\eta}^{\alpha}-v_{\eta}\right\rangle_{\mathcal{V}^{*} \times \mathcal{V}} \leq \int_{0}^{T}\left\langle\dot{v}_{\eta}^{\alpha}(s), v_{\eta}(s)-v_{\eta}^{\alpha}(s)\right\rangle_{V^{*} \times V} d s \\
& \quad+\int_{0}^{T}\left[j_{\alpha}\left(v_{\eta}(s)\right)-j_{\alpha}\left(v_{\eta}^{\alpha}(s)\right)\right] d s+\int_{0}^{T}\left\langle f_{\eta}(s), v_{\eta}^{\alpha}(s)-v_{\eta}(s)\right\rangle_{V^{*} \times V} d s .
\end{aligned}
$$


Passing to $\lim \sup$ as $\alpha \rightarrow 0$ in the last inequality by using (92)- 93 and (87), we obtain

$$
\limsup _{\alpha \rightarrow 0}\left\langle\mathcal{F}\left(v_{\eta}^{\alpha}\right), v_{\eta}^{\alpha}-v_{\eta}\right\rangle_{\mathcal{V}^{*} \times \mathcal{V}} \leq 0
$$

and since the operator $\mathcal{F}$ is bounded, hemicontinuous and monotone, we deduce that $\mathcal{F}$ is pseudomonotone (see, e.g., [19]), and keeping in mind (2), (87) and (95) we deduce the convergence 947).

STEP 4. In this step we consider the following variational problem.

Problem 16. Let $\eta \in L^{2}(0, T ; V)$. Find a function $v_{\eta} \in \mathcal{W}$ such that

$$
\begin{aligned}
\begin{aligned}
&\left\langle\dot{v}_{\eta}(s), w-v_{\eta}(s)\right\rangle_{V^{*} \times V}+\left(\mathcal{A}\left(\varepsilon\left(v_{\eta}(s)\right)\right), \varepsilon\left(w-v_{\eta}(s)\right)\right)_{\mathcal{Q}} \\
&+j_{\tau}(w)-j_{\tau}\left(v_{\eta}(s)\right) \geq\left\langle f_{\eta}(s), w-v_{\eta}(s)\right\rangle_{V^{*} \times V}, \\
& \forall w \in V, \text { a.e. } s \in(0, T),
\end{aligned} \\
\begin{aligned}
v_{\eta}(0)=v_{0} .
\end{aligned}
\end{aligned}
$$

We have the following existence and uniqueness result.

Lemma 17. Assume that 21)-(30) and (31)(ii) hold. Then problem (96)-97) has a unique solution $v_{\eta}$ which satisfies

$$
v_{\eta} \in L^{2}(0, T ; V) \cap C([0, T] ; H), \quad \dot{v}_{\eta} \in L^{2}\left(0, T ; V^{*}\right) .
$$

Proof. In view of (80), (77) we deduce that the function $v_{\eta}^{\alpha}$ satisfies

$$
\begin{aligned}
& \int_{0}^{T}\left\langle\dot{v}_{\eta}^{\alpha}(s), z(s)-v_{\eta}^{\alpha}(s)\right\rangle_{V^{*} \times V} d s \\
& \quad+\int_{0}^{T}\left(\mathcal{A}\left(\varepsilon\left(v_{\eta}^{\alpha}(s)\right)\right), \varepsilon\left(z(s)-v_{\eta}^{\alpha}(s)\right)\right)_{\mathcal{Q}} d s+\int_{0}^{T}\left[j_{\alpha}(z(s))-j_{\alpha}\left(v_{\eta}^{\alpha}(s)\right)\right] d s \\
& \geq \int_{0}^{T}\left\langle f_{\eta}(s), z(s)-v_{\eta}^{\alpha}(s)\right\rangle_{V^{*} \times V} d s, \quad \forall z \in L^{2}(0, T ; V) .
\end{aligned}
$$

Let $t \in(0, T)$ and $r>0$ be such that $t+r \in(0, T)$, and let $w \in V$. Then in (99) we put

$$
z(s)= \begin{cases}w & \text { for } s \in(t, t+r), \\ v_{\eta}(s) & \text { elsewhere }\end{cases}
$$

and pass to the limsup as $\alpha \rightarrow 0$, to obtain

$$
\begin{aligned}
& \frac{1}{r} \int_{t}^{t+r}\left\langle\dot{v}_{\eta}(s), w-v_{\eta}(s)\right\rangle_{V^{*} \times V} d s+\frac{1}{r} \int_{t}^{t+r}\left(\mathcal{A}\left(\varepsilon\left(v_{\eta}(s)\right)\right), \varepsilon\left(w-v_{\eta}(s)\right)\right)_{\mathcal{Q}} d s \\
& \quad+\frac{1}{r} \int_{t}^{t+r}\left[j_{\tau}(w)-j_{\tau}\left(v_{\eta}(s)\right)\right] d s \geq \frac{1}{r} \int_{t}^{t+r}\left\langle f_{\eta}(s), w-v_{\eta}(s)\right\rangle_{V^{*} \times V} d s, \quad \forall w \in V .
\end{aligned}
$$


Let $v_{\eta}$ be the function obtained in Lemma 14. Since $v_{\eta}^{\alpha}(0)=v_{\eta}(0)$, using (86) and passing to the limit as $r \rightarrow 0$ in the last inequality we deduce that the function $v_{\eta}$ is a solution of problem (96)-(97) such that (98) holds.

For the uniqueness, let $v_{1}, v_{2} \in \mathcal{W}$ be two functions satisfying (96)-(97). Setting, in (96), $\left(v_{\eta}, w\right)=\left(v_{1}, v_{2}\right)$ and $\left(v_{\eta}, w\right)=\left(v_{2}, v_{1}\right)$, and adding the two inequalities we get

$$
\begin{aligned}
\int_{0}^{T}\left\langle\dot{v}_{1}(s)-\right. & \left.\dot{v}_{2}(s), v_{1}(s)-v_{2}(s)\right\rangle_{V^{*} \times V} d s \\
& +\int_{0}^{T}\left(\mathcal{A}\left(\varepsilon\left(v_{1}(s)\right)\right)-\mathcal{A}\left(\varepsilon\left(v_{2}(s)\right)\right), \varepsilon\left(v_{1}(s)-v_{2}(s)\right)\right)_{\mathcal{Q}} d s \leq 0 .
\end{aligned}
$$

Keeping in mind (97) and (21), we deduce that

$$
\frac{1}{2}\left\|v_{1}(T)-v_{2}(T)\right\|_{H}^{2}+m_{\mathcal{A}}\left\|v_{1}-v_{2}\right\|_{L^{2}(0, T ; V)}^{2} \leq 0,
$$

which shows that $v_{1}=v_{2}$.

SteP 5. Let $\eta \in L^{2}(0, T ; V)$, let $v_{\eta}$ be the unique solution of problem (96)-(97), let $\beta_{\eta}$ be the unique solution of problem (64)-(66), let $\zeta_{\eta}$ be the unique solution of problem $(55)-(56)$, and define $\Lambda: L^{2}(0, T ; V) \rightarrow$ $L^{2}(0, T ; V)$ by

$$
\Lambda \eta(t)=\int_{0}^{t} v_{\eta}(s) d s+u_{0}, \quad \forall \eta \in L^{2}(0, T ; V), \forall t \in[0, T] .
$$

We have the following result.

Lemma 18. The operator $\Lambda$ has a unique fixed point $\eta^{*} \in L^{2}(0, T ; V)$.

Proof. Let $\eta_{1}, \eta_{2} \in L^{2}(0, T ; V)$, then by $(96)$ and 79 ,

$$
\begin{aligned}
& \int_{0}^{t}\left\langle\dot{v}_{\eta_{1}}(s)\right. \\
& \left.\quad-\dot{v}_{\eta_{2}}(s), v_{\eta_{1}}(s)-v_{\eta_{2}}(s)\right\rangle_{V^{*} \times V} d s \\
& \quad+\int_{0}^{t}\left(\mathcal{A}\left(\varepsilon\left(v_{\eta_{1}}(s)\right)\right)-\mathcal{A}\left(\varepsilon\left(v_{\eta_{2}}(s)\right)\right), \varepsilon\left(v_{\eta_{1}}(s)-v_{\eta_{2}}(s)\right)\right)_{\mathcal{Q}} d s \\
& \leq \int_{0}^{t} j_{\mathrm{ad}}\left(\beta_{\eta_{1}}(s), \eta_{1}(s), v_{\eta_{2}}(s)-v_{\eta_{1}}(s)\right) d s \\
& \quad-\int_{0}^{t} j_{\mathrm{ad}}\left(\beta_{\eta_{2}}(s), \eta_{2}(s), v_{\eta_{2}}(s)-v_{\eta_{1}}(s)\right) \\
& \quad+\int_{0}^{t}\left(\mathcal{B}\left(\varepsilon\left(\eta_{1}(s)\right), \zeta_{\eta_{1}}(s)\right)-\mathcal{B}\left(\varepsilon\left(\eta_{2}(s)\right), \zeta_{\eta_{2}}(s)\right), \varepsilon\left(v_{\eta_{2}}(s)-v_{\eta_{1}}(s)\right)\right)_{\mathcal{Q}} d s .
\end{aligned}
$$


Using (97), (21)-(22), (38) and (67)(ii), we obtain

$$
\begin{aligned}
\left\|v_{\eta_{1}}(t)-v_{\eta_{2}}(t)\right\|_{H}^{2}+ & \int_{0}^{t}\left\|v_{\eta_{1}}(s)-v_{\eta_{2}}(s)\right\|_{V}^{2} d s \\
\leq & c \int_{0}^{t}\left\|\beta_{\eta_{1}}(s)-\beta_{\eta_{2}}(s)\right\|_{L^{2}\left(\Gamma_{3}\right)}\left\|v_{\eta_{1}}(s)-v_{\eta_{2}}(s)\right\|_{V} d s \\
& +c \int_{0}^{t}\left\|\eta_{1}(s)-\eta_{2}(s)\right\|_{V}\left\|v_{\eta_{1}}(s)-v_{\eta_{2}}(s)\right\|_{V} d s \\
& +c \int_{0}^{t}\left\|\zeta_{\eta_{1}}(s)-\zeta_{\eta_{2}}(s)\right\|_{V}\left\|v_{\eta_{1}}(s)-v_{\eta_{2}}(s)\right\|_{V} d s
\end{aligned}
$$

We then use the inequality

$$
\lambda \delta \leq \lambda^{2}+\frac{1}{4} \delta^{2}, \quad \forall \lambda, \delta \in \mathbb{R} .
$$

We deduce that

$$
\begin{aligned}
\int_{0}^{t}\left\|v_{\eta_{1}}(s)-v_{\eta_{2}}(s)\right\|_{V}^{2} d s & \leq c \int_{0}^{t}\left\|\beta_{\eta_{1}}(s)-\beta_{\eta_{2}}(s)\right\|_{L^{2}\left(\Gamma_{3}\right)}^{2} d s \\
& +c \int_{0}^{t}\left\|\eta_{1}(s)-\eta_{2}(s)\right\|_{V}^{2} d s+c \int_{0}^{t}\left\|\zeta_{\eta_{1}}(s)-\zeta_{\eta_{2}}(s)\right\|_{L^{2}(\Omega)}^{2} d s .
\end{aligned}
$$

Therefore, by (68) and 58),

$$
\int_{0}^{t}\left\|v_{\eta_{1}}(s)-v_{\eta_{2}}(s)\right\|_{V}^{2} d s \leq c \int_{0}^{t}\left\|\eta_{1}(s)-\eta_{2}(s)\right\|_{V}^{2} d s .
$$

Now, using (100), we obtain

$$
\left\|\Lambda \eta_{1}(t)-\Lambda \eta_{2}(t)\right\|_{V}^{2} \leq c \int_{0}^{t}\left\|v_{\eta_{1}}(s)-v_{\eta_{2}}(s)\right\|_{V}^{2} d s
$$

which, together with 101, implies that

$$
\left\|\Lambda \eta_{1}(t)-\Lambda \eta_{2}(t)\right\|_{V}^{2} \leq c \int_{0}^{t}\left\|\eta_{1}(s)-\eta_{2}(s)\right\|_{V}^{2} d s, \quad \forall t \in[0, T] .
$$

Reiterating the last inequality $n$ times, we infer that

$$
\left\|\Lambda \eta_{1}-\Lambda \eta_{2}\right\|_{L^{2}(0, T ; V)}^{2} \leq \frac{(c T)^{n}}{n !}\left\|\eta_{1}-\eta_{2}\right\|_{L^{2}(0, T ; V)}^{2},
$$

which implies that, for $n$ sufficiently large, a power $\Lambda^{n}$ of $\Lambda$ is a contraction in the Banach space $L^{2}(0, T ; V)$. Hence $\Lambda$ has a unique fixed point $\eta^{*} \in$ $L^{2}(0, T ; V)$. 
Now, we have all the ingredients to prove Theorem 7 . Let $\Lambda$ be the operator defined by (100), let $\eta^{*}$ be the fixed point of $\Lambda$, let $\beta=\beta_{\eta^{*}}$ be the unique solution of problem (64)-(66) corresponding to $\eta^{*}$, let $\zeta=\zeta_{\eta^{*}}$ be the unique solution of problem (55)-(56), and let $u:[0, T] \rightarrow V$ be the displacement field defined by

$$
u(t)=\eta^{*}(t)=\int_{0}^{t} v_{\eta^{*}}(s) d s+u_{0}, \quad \forall t \in[0, T],
$$

where $v_{\eta^{*}}$ is the unique solution of problem (96)-(97) corresponding to $\eta^{*}$. We conclude by (96)-(97), (64)-(66), (55)-(56) and $(102)$ that $\{u, \beta, \zeta\}$ is a solution of problem (46)-(51). Moreover, the regularity (52)-(54) follows from (67), (57), (98) and (102). The uniqueness of the solution is a consequence of the uniqueness of the fixed point of the operator $\Lambda$ and of the uniqueness of the solution of problems (64)-(66), (55)- $-(56)$ and (96)- (97). Finally, it is easy to see, in this case, that the function $\sigma$ defined by (6) satisfies

$$
\sigma \in L^{2}(0, T ; \mathcal{Q}), \quad \operatorname{Div} \sigma \in L^{2}\left(0, T ; V^{*}\right) .
$$

\section{References}

[1] V. Barbu, Semigroups and Differential Equations in Banach Spaces, Noordhoff, Leyden, 1976.

[2] V. Barbu, Optimal Control of Variational Inequalities, Pitman, Boston, 1984.

[3] M. Campo, J. R. Fernández and K. L. Kuttler, Analysis of a dynamic frictional contact problem with damage, Finite Elements Anal. Design 45 (2009), 659-674.

[4] O. Chau, J. R. Fernández, W. Han and M. Sofonea, Variational and numerical analysis of a dynamic frictionless contact problem with adhesion, J. Comput. Appl. Math. 156 (2003), 127-157.

[5] M. Cocu and R. Rocca, Existence results for unilateral quasistatic contact problems with friction and adhesion, Math. Model. Numer. Anal. 34 (2000), 981-1001.

[6] G. Duvaut et J.-L. Lions, Les Inéquations en Mécanique et en Physique, Dunod, Paris, 1972.

[7] J. R. Fernández, M. Shillor and M. Sofonea, Analysis and numerical simulations of a dynamic contact problem with adhesion, Math. Computer Modelling 37 (2003), $1317-1333$.

[8] M. Frémond, Équilibre des structures qui adhèrent à leur support, C. R. Acad. Sci. Paris Sér. II 295, (1982), 913-916.

[9] M. Frémond, Adhérence des solides, J. Méc. Théor. Appl. 6 (1987), 383-407.

[10] M. Frémond, Non-Smooth Thermomechanics, Springer, Berlin, 2002.

[11] M. Frémond and B. Nedjar, Damage in concrete: the unilateral phenomenon, Nuclear Engrg. Design 156 (1995), 323-335.

[12] M. Frémond and B. Nedjar, Damage, gradient of damage and principle of virtual work, Int. J. Solids Structures 33 (1996), 1083-1103.

[13] J. Han and S. Migórski, A quasistatic viscoelastic frictional contact problem with multivalued normal compliance, unilateral constraint and material damage, J. Math. Anal. Appl. 443 (2016), 57-80. 
[14] W. Han and M. Sofonea, Quasistatic Contact Problems in Viscoelasticity and Viscoplasticity, Stud. Adv. Math. 30, Amer. Math. Soc. and Int. Press, Providence, RI, 2002.

[15] W. Han and M. Sofonea, On a dynamic contact problem for elastic-visco-plastic materials, Appl. Numer. Math. 57 (2007), 498-509.

[16] L. Jianu, M. Shillor and M. Sofonea, A viscoelastic bilateral frictionless contact problem with adhesion, Appl. Anal. 80 (2001), 233-255.

[17] Y. Li and Z. Liu, A quasistatic contact problem for viscoelastic materials with friction and damage, Nonlinear Anal. 73 (2010), 2221-2229.

[18] Y. Li, S. Migórski and J. Han, A quasistatic frictional contact problem with damage involving viscoelastic materials with short memory, Math. Mech. Solids 21 (2016), $1167-1183$.

[19] J.-L. Lions, Quelques méthodes de résolution des problèmes aux limites non linéaires, Dunod et Gauthier-Villars, 1969.

[20] J. Nečas and I. Hlavaček, Mathematical Theory of Elastic and Elastoplastic Bodies: An Introduction, Elsevier, Amsterdam, 1981.

[21] M. Raous, L. Cangémi and M. Cocu, A consistent model coupling adhesion, friction and unilateral contact, Comput. Methods Appl. Engrg. 177 (1999), 383-399.

[22] M. Shillor, M. Sofonea and J. J. Telega, Models and Analysis of Quasistatic Contact, Springer, Berlin, 2004.

[23] M. Sofonea, W. Han and M. Shillor, Analysis and Approximations of Contact Problems with Adhesion or Damage, Pure Appl. Math. 276, Chapman \& Hall/CRC Press, Boca Raton, FL, 2006.

[24] P. Suquet, Plasticité et homogénéisation, thesis, Univ. of Paris VI, 1982.

[25] A. Touzaline, A quasistatic bilateral contact problem with adhesion and friction for viscoelastic materials, Comment. Math. Univ. Carolin. 51 (2010), 85-97.

Abderrezak Kasri

Département de Mathématiques

Université 20 Août 1955 - Skikda

B.P. 26, Route El-Hadaiek

Skikda, Algeria

E-mail: kariabdezak@gmail.com
Arezki Touzaline Laboratoire de Systèmes Dynamiques Faculté de Mathématiques

USTHB

B.P. 32, El Alia

Bab-Ezzouar, 16111 Alger, Algeria

E-mail: ttouzaline@yahoo.fr 
